\title{
Multi-State VALBOND for Atomistic Simulations of Hypervalent Molecules, Metal Complexes and Reactions
}

\author{
Maurus H. Schmid, ${ }^{\dagger, \dagger}$ Akshaya Kumar Das,, $\boldsymbol{\Phi}^{\dagger}$ Clark R. Landis, ${ }^{* \dagger}$ and Markus \\ Meuwly*,
}

These authors contributed equally, Department of Chemistry, University of Wisconsin-Madison, 1101 University Avenue, Madison, Wisconsin 53706, United States, and Department of Chemistry, University of Basel, Klingelbergstrasse 80, 4056 Basel, Switzerland

E-mail: landis@chem.wisc.edu; m.meuwly@unibas.ch

February 27, 2018

\begin{abstract}
The implementation, validation and application of the Multi-State VALBOND (MSVALBOND) method for transition metal-containing and hypervalent molecules is presented. This approach is particularly suited for molecules with unusual shapes and systems that need to be described by a superposition of resonance structures, each of which satisfies the octet rule. The implementation is based on the original VALBOND force field and allows to smoothly switch between resonance structures, each of which

${ }^{*}$ To whom correspondence should be addressed

${ }^{\dagger}$ These authors contributed equally

$\ddagger$ University of Wisconsin-Madison

"University of Basel
\end{abstract}


can be characterized by its own force field, including varying charge distributions, and coupling terms between the states. The implementation conserves total energy for simulations in the gas phase and in solution and is applied to a number of topical systems. For the small hypervalent molecule $\mathrm{ClF}_{3}$ the barrier for pseudorotation is found to be $4.3 \mathrm{kcal} / \mathrm{mol}$ which compares favourable with the experimentally measured value of $4.8 \mathrm{kcal} / \mathrm{mol}$. A transition metal-containing complex is cisplatin, characterized by 6 resonance states, for which the vibrational spectrum is found to be in good agreement with experiment. Finally, umbrella sampling simulations of the $\mathrm{S}_{\mathrm{N}} 2$ reaction $\mathrm{BrMe}+\mathrm{Cl}^{-} \rightarrow \mathrm{Br}^{-}+\mathrm{MeCl}$ in solution yields a barrier height of $24.6 \mathrm{kcal} / \mathrm{mol}$, in good agreement with experiment $(24.7 \mathrm{kcal} / \mathrm{mol})$.

\section{Introduction}

Atomistic simulations are a powerful means to investigate the structure and dynamics of complex systems, such as proteins in solution. Over the past 10 to 15 years the quality of such simulations has continuously improved not least due to the availability of increasingly accurate but empirical energy functions. Initially, their development focused on the chemical structure and dynamics of macromolecules, including peptides and proteins. ${ }^{1-7}$ More recently, considerable progress has been made by incorporating effects due to anisotropic charge distributions (multipolar electrostatics) ${ }^{8-15}$ and polarizability. ${ }^{16-18}$ Such approaches have been very successful for applications in thermodynamics, computational spectroscopy or for investigating ligand binding to proteins.

However, when the complexity of the electronic structure of the compounds considered increases, as is the case for halogenated molecules or systems involving transition metals (TMs), even such more refined energy functions have their limitations. The situation is particularly demanding for metal-containing systems. Ideally, it would be possible to investigate the dynamics of such systems using mixed quantum mechanical/molecular mechanics molecular 
dynamics simulations (QM/MM MD). ${ }^{19-21}$ In $\mathrm{QM} / \mathrm{MM}$ the total system is divided into a (typically small) region for which the energy is calculated quantum mechanically - i.e. from electronic structure methods - and an environment which is treated with a conventional force field. However, due to the $N^{3}$ scaling of the secular determinants that need to be diagonalized, where $N$ is the number of basis functions used in the QM part, routine applications can only be envisaged for systems including several tens of heavy atoms in the quantum region (fewer if metals are involved), and by using density functional theory (DFT) to describe the energetics. ${ }^{22}$ Typical simulation times that can be afforded under such circumstances are on the order of several 10 picoseconds which is usually too short for reactions with appreciable activation energies. ${ }^{23}$ An exception to this are semiempirical quantum methods, such as density functional tight binding (DFTB) ${ }^{24,25}$

Hence, if the energetics and dynamics of reactive processes involving metals needs to be characterized, alternative methods to describe the energetics of the systems are required. Transition metals play important roles for processes including electron transport, oxidation reactions, or oxygen transport. ${ }^{26-29}$ Also, metal complexes find broad applications for both homogeneous and heterogeneous catalysis in organic synthesis. ${ }^{30}$ However, atomistic simulations using empirical force fields for metals in particular and hypervalent compounds in general pose particular challenges. The difficulty arises due to variable coordination number (as exemplified for $\mathrm{Co}(\mathrm{CO})_{4}, \mathrm{Cr}(\mathrm{CO})_{5}$, or $\left.\mathrm{Cr}(\mathrm{CO})_{6}\right)$, indistinct topology $(\pi$ ligands can bind to metal in different ways), the different oxidation states (eg. $\mathrm{Cu}(\mathrm{I})$ and $\mathrm{Cu}(\mathrm{II})$ shows tetrahedral and square planar geometries respectively) $)^{25,31-33}$ and the coupling of electronic and vibrational degrees of freedom (i.e. Jahn- Teller distortion).

In the past, several methods reminiscent of empirical force fields have been developed to treat transition metal complexes and organometallics. The main idea underlying metal force fields is to extend a conventional force field with one or several additional terms to capture specific 
properties of TMs. These include large angle distortions including bending potentials with several minima, the effects due to $d$-electrons (such as the Jahn-Teller effect) or binding of TMs to the $\pi$-electron cloud (as in ferrocenes). As an example, the difference in bonding of axial versus equatorial ligands to a TM can be described by two different parameter sets for the two types of ligands. On the other hand, such an effect can be captured within the framework of ligand field stabilization as it has been done in ligand field molecular mechanics (LFMM). ${ }^{34}$ Such an approach is also capable of capturing stereo-electronic effects of partially filled $d$-orbitals in coordination compounds.

Alternatively, the VALBOND force field ${ }^{35-38}$ field, which is based on valence bond theory and hybrid orbital strength functions, is able to describe the angular distortions for a wide range around the metal center. According to the VALBOND formalism, ${ }^{35-38}$ hypervalent molecules are molecules that exceed an octet electron count (e.g. $\mathrm{ClF}_{3}$ or $\mathrm{SF}_{6}$ ). Hypervalent molecules i.e. molecules where certain atoms have more than the formal 8 electrons, are treated by using resonance structures that formally fulfill the octet rule (or duodectet rule). For metal complexes which primarily use the $n$ s and $(n-1) \mathrm{d}$ metal orbitals in forming bonds, electron counts in excess of 12 electrons are considered hypervalent. ${ }^{39}$ Therefore, most common transition metal complexes are considered to be hypervalent and approaches such as VALBOND or VALBOND-TRANS are useful to follow the structural dynamics of TM-complexes. ${ }^{33,37,38,40-42}$ In the original VALBOND formalism hypervalent molecules were represented by combinations of 2-center-2-electron and 3-center-4-electron bonds; in the localized framework 3-center-4-electrons comprise resonance among two primary Lewis-like configurations. This approach was successful for hypervalent first row molecules (such as $\left.\mathrm{ClF}_{3}\right)$ as well as TM-complexes. ${ }^{35-38}$ Originally, it was only an angle potential. Later it was extended to model the trans-influence in metal complexes by introducing two energy and distance dependent penalty functions to the original VALBOND and the method called VALBOND-TRANS. ${ }^{40}$ 
Besides the structural characterization of metal complexes and the dynamics around them, studying chemical reactions is fundamental in chemistry and biology. Since chemical reactions involve the breaking and making of chemical bonds, conventional force fields can not be used for such applications. However, over the years several methods such as reactive force field (ReaxFF), ${ }^{43}$ empirical valence bond $(\mathrm{EVB})^{44}$ and its multi-state variant MS-EVB which was primarily used to investigate proton transport, ${ }^{45,46}$ adiabatic reactive molecular dynamics $(\mathrm{ARMD})^{47,48}$ and multi-state adiabatic reactive molecular dynamics (MS-ARMD) ${ }^{49}$ have been developed to follow chemical reactions in the gas phase and in solution. Here, we set out to generalize VALBOND ${ }^{35-38}$ by developing a comprehensive framework approach based on resonance among structures that fulfill the octet rule. This new approach treats angular degrees of freedom within a valence bond description and further includes chemical bonds, dihedrals and van der Waals contributions by flexible parametrizations of individual resonance structures ("states").

The new method, referred to as multi-state VALBOND (MS-VALBOND), as discussed in the present manuscript explicitly treats differences in charge distributions between the resonance structures/states and allows bond breaking and bond formation. It is therefore a method that allows to investigate chemical reactions involving hypervalent and transition metals at approximately the speed of empirical force fields. The method was implemented in $\mathrm{CHARMM}^{5}$ and was tested by applying it to three different model cases including the hypervalent molecule $\mathrm{ClF}_{3}$, a prototypical metal complex (cisplatin) and the chemical reaction of $\mathrm{BrMe}+\mathrm{Cl}^{-} \rightarrow \mathrm{Br}^{-}+\mathrm{MeCl}$. 


\section{Computational Methods}

Two models of bonding in hypervalent molecules have been proposed previously: the original Pauling approach expanded the valency by using extravalent orbitals (e.g., d-orbitals for main group elements) to accommodate electrons that exceed the octet. Alternatively, hypervalency has been described using an ionic resonance model in which linear arrangements of three atoms undergo three-center/four-electron (3c/4e) bonding. The latter model, which is supported by modern analyses of electronic structure, ${ }^{50}$ was originally presented in the context of MO theory by Pimentel ${ }^{51}$ and Rundle. ${ }^{52}$ However, Coulson ${ }^{53}$ showed that the VB description involving resonance between octet-conforming configurations [ Y: XY $\leftrightarrow$ YX :Y ] essentially is equivalent to the MO density distribution. The dominance of such resonance structures is further supported by NBO analyses ${ }^{39,54}$ and ab initio VB computations. ${ }^{55}$ In the NBO framework, the 3c/4e bond corresponds to a donor-acceptor interaction comprising donation of a lone pair of electrons from $\mathrm{Y}$ into the $\mathrm{X}-\mathrm{Y}$ antibond.

The present approach addresses several limitations of empirical force fields for hypervalent molecules in general and metal-containing complexes in particular. First, the angular terms AXB (where A and B are atoms of ligand and X is a metal or hypervalent center) are treated by VALBOND which enables description of geometries for the bending degrees of freedom around metal centers. Secondly, each relevant resonance structure is treated as a different connectivity ("state"). The total energy of the system is described by the Hamiltonian

$$
H=\left[\begin{array}{cccc}
H_{I, I} & H_{I, I I} & \cdots & H_{I, n} \\
H_{I I, I} & H_{I I, I I} & \cdots & H_{I I, n} \\
\vdots & \vdots & \ddots & \vdots \\
H_{n, I} & H_{n, I I} & \cdots & H_{n, n}
\end{array}\right]
$$


where the diagonal elements are the force fields of each connectivity/resonance structure $H_{i i}$ and the off-diagonal elements are the couplings $H_{i j}$ between them. This formulation is akin to an the empirical valence bond (EVB) ansatz. ${ }^{44,56}$ For a single molecule this offers the opportunity to treat the same chemical bond with different parametrizations so that effects such as alteration between single, double or partial-double bonds of one and the same bond depending on the environment can be captured. This information would be available in the strength (force constant $k$ ) and equilibrium bond length $r_{e}$. It should also be possible to represent different oxidation states, e.g. $\mathrm{Cu}(\mathrm{I})$ and $\mathrm{Cu}(\mathrm{II}) .{ }^{25}$ Furthermore, the transinfluence $^{40}$ (known from metal-complexes) which was incorporated in VALBOND-TRANS, can be readily included. This can be achieved through suitable parametrization of the offdiagonal elements which is described further below.

Diagonalization of $\mathbf{H}$ yields $n$ eigenvalues and eigenvectors and the lowest eigenvalue $E_{0}$ with eigenvector $\overrightarrow{\nu_{0}}=\left(\nu_{0}^{(\mathrm{I})}, \nu_{0}^{(\mathrm{II})}, \nu_{0}^{(\mathrm{III})}, \ldots ., \nu_{0}^{(\mathrm{N})}\right)$ is used for propagation of the system.

$$
E\left(\Psi_{t o t}\right)=\left\langle\Psi_{t o t}|H| \Psi_{t o t}\right\rangle
$$

The contribution of state $i$ towards the ground state energy $E_{0}$ is $p_{i}=\nu_{0}^{i} \nu_{0}^{i}$. As all elements in $\mathbf{H}$ depend on internal coordinates, transitions between states along a trajectory can be followed by considering the populations $p_{i}$ of the states.

\subsection{Parametrization of the Diagonal Elements}

Each diagonal term describes a resonance structure. Thus each diagonal matrix element is treated and parametrized independently (see section 3.1). The diagonal element $\left(H_{i i}(\mathbf{X})\right)$ represents the energy of the $i^{\text {th }}$ resonance structure for a given configuration $\mathbf{X}$. Bonds which break and form or which change dissociation energy or equilibrium structure between the 
states are described by Morse bonds (see equation 3).

$$
V_{M}(r)=D_{e}\left(\left(1-e^{-\beta\left(r-r_{e}\right)}\right)^{2}-1\right)
$$

In equation $3, r$ is the separation between the atoms in the bond, $r_{\mathrm{e}}$ is the equilibrium distance, $D_{\mathrm{e}}$ is the dissociation energy of the bond, and $\beta$ describes the steepness of the short range part of the potential. Angles are described by the VALBOND formalism ${ }^{35}$ which is rooted in valence bond theory. ${ }^{57}$

Similarly, following previous work, ${ }^{58}$ intramolecular van der Waals interactions are also described by Morse potentials but with small dissociation energy. This functional form has been shown to provide a better representation of the energy, especially at the short distances on the repulsive side of the energy function. Resonance structures for 3-center-4-electron bonds nearly always involve short separation in which distances (e.g., the $\mathrm{F}_{\mathrm{D}}-\mathrm{Cl}_{\mathrm{A}}$ distance in resonance structure I in Figure 1) are on the repulsive side of the non-bond potential. The non-bonded parameters of individual atoms were obtained by fitting Morse potential to the ab initio potential energy curve obtained along the $\mathrm{Cl}-\mathrm{He}$ and $\mathrm{F}-\mathrm{He}$ bond. Helium was used as a probe because it interacts negligibly with its environment and provides a meaningful starting point for further refinement of the parameters (see Figure S1). To describe the non-bonded interactions between non-identical atoms combination rules akin to the Lorentz-Berthelot rules are used: $D_{i j}=\frac{D_{i}+D_{j}}{2}, \beta_{i j}=\frac{\beta_{i}+\beta_{j}}{2}$ and $r^{e}{ }_{i j}=\frac{r_{i}^{e}+r_{j}^{e}}{2}$. It would also be possible to represent the van der Waals interactions with a conventional 12-6 form or an exponential decay with a long-range dispersion term.

To illustrate the application of the formalism the example of $\mathrm{ClF}_{3}$ is considered, see Figure 1. The number of bonds and resonance structures required to describe the system depends on the number of valence electrons. To satisfy the octet rule one must only consider covalent 


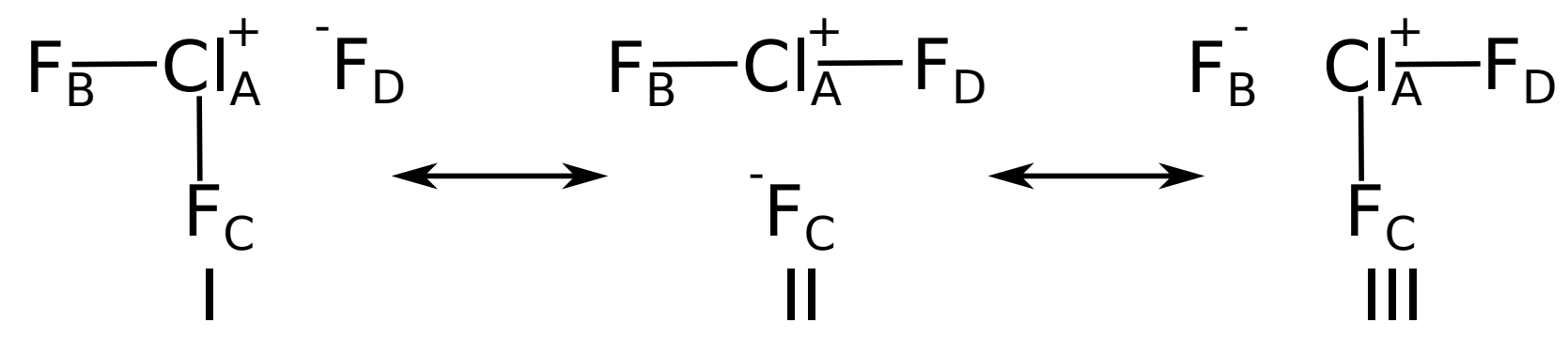

Figure 1: The three resonance structures, I to III, for hypervalent $\mathrm{ClF}_{3}$ such that each structure fulfills the octet rule. In resonance structure $\mathrm{I}$, two fluorine atoms $\left(\mathrm{F}_{\mathrm{B}}\right.$ and $\left.\mathrm{F}_{\mathrm{C}}\right)$ form a bond to the Chlorine atom $\mathrm{Cl}_{\mathrm{A}}$ to give octet-consistent $\mathrm{ClF}_{2}^{+}$. The third atom, $\mathrm{F}_{\mathrm{D}}$, is the octet-consistent fluoride anion $\mathrm{F}^{-}$. At the equilibrium T-shaped geometry, the F-Cl-F bond angles of the dominant resonance structures are close to $90^{\circ}$, corresponding to high p-character in the $\mathrm{Cl}-\mathrm{F}$ bonds of the $\mathrm{ClF}_{2}^{+}$fragments.

i.e. 2-center-2-electron bonds. In $\mathrm{ClF}_{3}$, three fluorine atoms are connected to the central chlorine atom. The chloride radical $\mathrm{Cl}^{\bullet}$ and 3 fluorine radicals $\mathrm{F}^{\bullet}$ contain $28(4 \times 7)$ electrons. The fully electron saturated (ionic) state formed by $\mathrm{F}^{-}$and $\mathrm{Cl}^{-}$would contain 32 electrons, hence 4 electrons are involved in bonding. Two electrons per covalent bond leads to $N=2$ bonds per resonance structure. As a total of $K=3$ bonds can be accommodated in $\mathrm{ClF}_{3}$, there are $\left(\begin{array}{l}N \\ K\end{array}\right)=\left(\begin{array}{l}2 \\ 3\end{array}\right)=3$ possible states (resonance structures) that need to be described, see Figure 1.

The force field description for resonance structure I consists of two bonds, which are represented each as a Morse potential with a set of fitted parameters and a VALBOND angle energy.

$$
V(I)=V_{M}\left(r_{\mathrm{AB}}\right)+V_{M}\left(r_{\mathrm{AC}}\right)+V\left(\alpha_{\mathrm{BAC}}\right)
$$

The angle $\alpha_{\mathrm{BAC}}$ between these bonds with the atom $\mathrm{A}$ at its apex is treated using valence bond theory. Within valence bond theory, for central atom A with $s p^{m} d^{n}$ hybridization the energy depends only on the force constant $k \cdot{ }^{57,59,60}$

$$
V\left(\alpha_{\mathrm{BAC}}, k, m, n\right)=k\left(S^{\max }-S(\alpha)\right)
$$


The general expression for the strength of hybrid orbitals $S$ as derived by Pauling ${ }^{60}$ is

$$
S(\alpha)=S^{\max } \sqrt{1-\frac{1-\sqrt{1-\Delta^{2}}}{2}}
$$

where $S^{\max }$ is the maximum of the strength function of hybrid orbitals and $\Delta$ is the overlap integral and their functional forms are given by

$$
\begin{gathered}
S^{\max }=\sqrt{\frac{1}{1+m+n}}(1+\sqrt{3 m}+\sqrt{5 n}) \\
\Delta=\frac{1}{1+m+n}\left(1+m \cos \alpha+\frac{n}{2}\left(3 \cos ^{2} \alpha-1\right)\right)
\end{gathered}
$$

In VALBOND, the overlap $(\Delta)$ between two hybrid (bond-)orbitals $\left(\psi_{1}, \psi_{2}\right)$ is expressed as a function of the hybridizations $m$ and $n$ which are the formal $p$ and $d$ hybridizations. The force constant $k$ can be chosen to best describe the bending frequency.

The nonbonded interactions in $\mathrm{ClF}_{3}$ for resonance structure I (see Figure 1) are described by

$$
\begin{aligned}
V(r)= & V_{M}\left(r_{\mathrm{AD}} ; r_{\mathrm{e}, \mathrm{AD}}, D_{\mathrm{e}, \mathrm{AD}}, \beta_{\mathrm{AD}}\right)+ \\
& V_{M}\left(r_{\mathrm{BD}} ; r_{\mathrm{e}, \mathrm{BD}}, D_{\mathrm{e}, \mathrm{BD}}, \beta_{\mathrm{BD}}\right)+ \\
& V_{M}\left(r_{\mathrm{CD}} ; r_{\mathrm{e}, \mathrm{CD}}, D_{\mathrm{e}, \mathrm{CD}}, \beta_{\mathrm{CD}}\right)
\end{aligned}
$$

with cyclical permutations for the other two states II and III. The parameters for these diagonal matrix elements (2c-2e bonds) are fitted for the resonance structure, i.e. they are fitted for $\mathrm{ClF}_{2}^{+}$in the case of $\mathrm{ClF}_{3}$. 


\subsection{Parametrization of the Off-Diagonal Elements}

The off-diagonal terms describe the coupling between states $i$ and $j$. Within the present context, these are stabilizations between two resonance structures. The resonance between structures $i$ and $j$ are modeled as a scaled Morse potential depending on the angle between the bonds that are modified upon resonance from one to another resonance structure (see Figure 2) according to

$$
V(i \rightarrow j)=\left(\cos \left(\omega_{\mathrm{CAD}}\right)-1\right)^{2} * V_{M}\left(r_{\mathrm{AD}}\right)
$$

The reverse resonance $(j \rightarrow i)$ is modeled along the same line

$$
V(j \rightarrow i)=\left(\cos \left(\omega_{\mathrm{CAD}}\right)-1\right)^{2} * V_{M}\left(r_{\mathrm{AC}}\right)
$$

In order to maintain the symmetry of the Hamiltonian, the final off-diagonal element is

$$
V_{i j}=V_{j i}=V(i \rightarrow j)+V(j \rightarrow i)
$$

The off-diagonal Morse parameters are then fitted to reproduce reference electronic structure data, vide infra. Effects such as the trans influence ${ }^{61}$ or Jahn-Teller distortion ${ }^{62}$ (coupling of vibrational and electronic degrees of freedom) can be readily included by suitably parametrizing the off-diagonal elements that reflect these effects. 

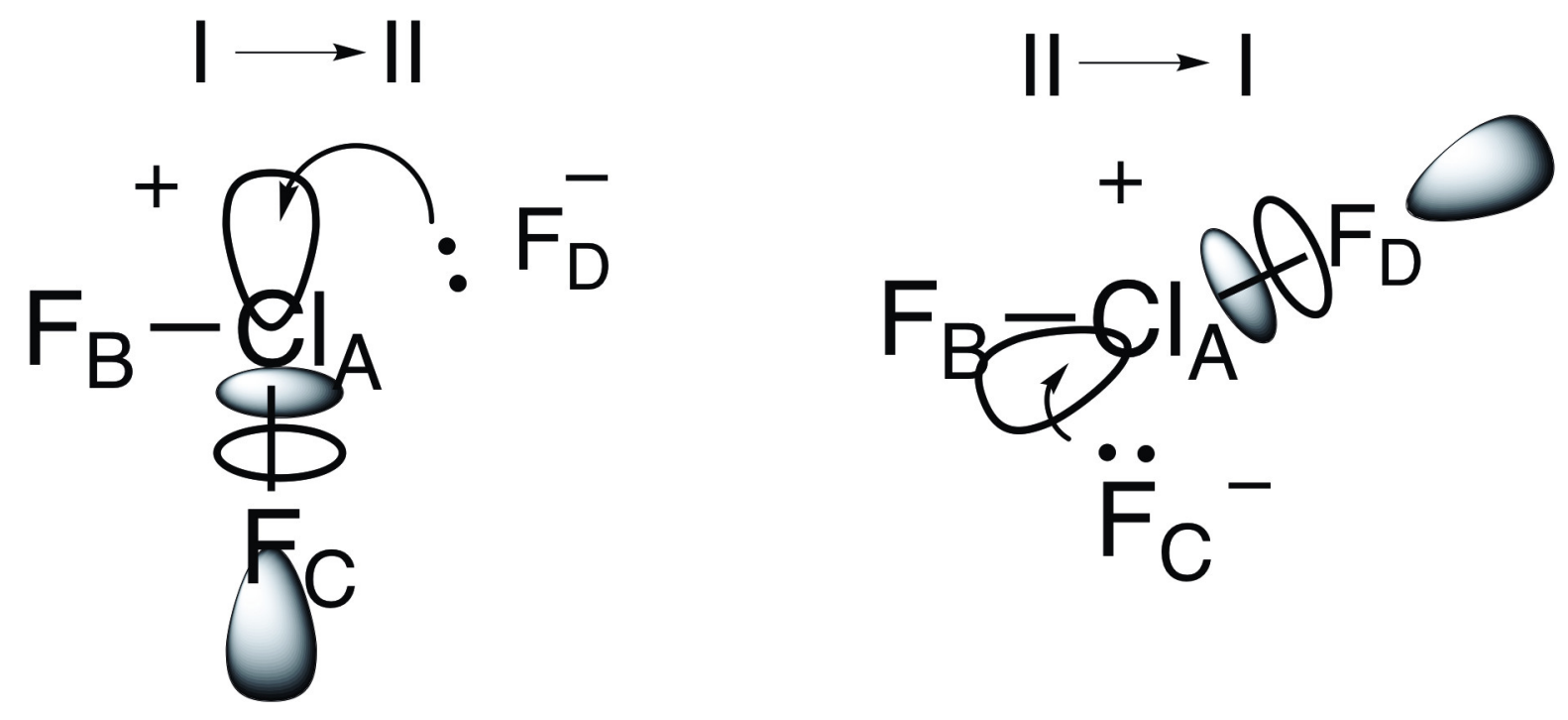

Figure 2: Resonance structures I and II, comprising ionic $[\mathrm{BAC}]^{+} \mathrm{D}^{-}$and $[\mathrm{BAD}]^{+} \mathrm{C}^{-}$Lewis configurations, respectively, undergo resonance via donation:acceptor interaction of $\mathrm{F}^{-}$lone pairs with vacant $\mathrm{Cl}-\mathrm{F}$ anti bonds. For example, resonance structure $\mathrm{I}$ is transformed by donation of a lone pair on the anion $\mathrm{F}_{\mathrm{D}}$ into the $\left(\mathrm{Cl}_{\mathrm{A}} \mathrm{F}_{\mathrm{C}}\right)$ anti-bond; such donor-acceptor interactions are the equivalent of "arrow-pushing" depictions of resonance. Shaded and empty circles indicate the lobes of the anti-bonding orbital and, for clarity, the molecular geometry shown is slightly distorted from the equilibrium T-shape. The strength of the donor-acceptor interaction depends on the overlap of donor and acceptor orbitals, hence, on the molecular geometry. At the geometry depicted above, the $\mathrm{I} \rightarrow \mathrm{II}$ overlap is substantially larger than the $\mathrm{II} \rightarrow \mathrm{I}$ overlap due to the more co-linear arrangement of the lone pair hybrid and the anti bonding orbital.

For $\mathrm{ClF}_{3}$ the resonance between states $i$ and $j$ is modelled as the donation of the free electron pair into the anti-bonding orbital of the bond that is broken. ${ }^{39}$ For the resonance between states I and II, the AC bond is broken while bond AD is formed. Considering $p$ orbitals, the donation of the free electron pair from $\mathrm{F}_{\mathrm{D}}$ into the anti-bond depends on the angle between the three atoms A, C, and D. Scaling of the off-diagonal term (see Figure 3) is achieved with the term $\left(\cos \left(\alpha_{\mathrm{DAC}}\right)-1\right)^{2}$ which switches between a minimal amplitude $\left(\omega_{\mathrm{CAD}}=0^{\circ}\right)$ and a maximum amplitude $\omega_{\mathrm{CAD}}=180^{\circ}$, thus modeling the expected donor-acceptor overlaps. 


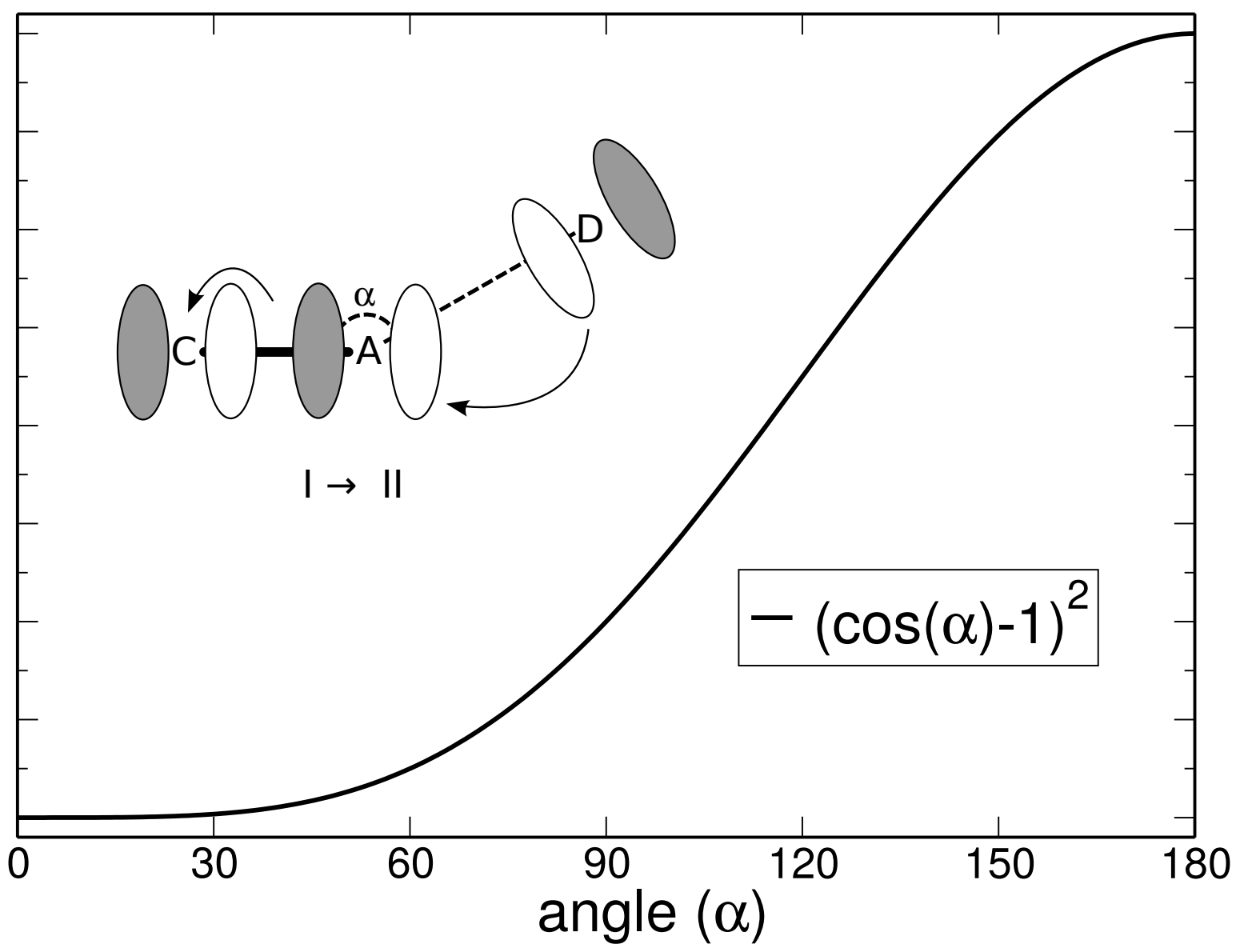

Figure 3: Considering $p$ orbitals, the donation into the anti-bond depends on the angle between the three involved atoms. For this, the off-diagonal term is scaled with $\left(\cos \left(\alpha_{\mathrm{DAC}}\right)-\right.$ $1)^{2}$ which is minimal at an angle of $0^{\circ}$ at maximal at $180^{\circ}$ where $\alpha$ is the angle between two overlapping orbitals.

\subsection{Solute-Solvent Electrostatic Interaction in MS-VALBOND}

Because including all solvent molecules into the description of the states would be computationally demanding, it is preferable to perform a mixed MS-VALBOND/MM simulation in the spirit of a mixed quantum mechanical/molecular mechanics (QM/MM) approach. Since for each resonant structure included in the simulation the charge distribution typically differs, electron flow (or charge reorganization) needs to be followed during transitions between states. The different charge distributions characterizing each individual state lead to different solvent-solute interactions for each state and the forces need to be determined 
such that total energy is conserved. This concerns primarily the electrostatic interactions $E_{\text {elstat }}=\frac{Q_{\text {solv }} q_{k}}{|R-r|}$ where the $Q_{\text {solv }}$ are the static solvent charges, $q_{k}$ are the solute charges of atom $k$ which fluctuate due to the different resonant structures, and $|R-r|$ is the separation between the MM-charge $Q$ and the MS-VALBOND (state-dependent charge) $q$.

For most of the structures along an MD trajectory the charge $q_{k}$ on a particular atom $k$ will be that of one specific state $i$ with weight $w_{i} \approx 1$ and all other weights $w_{j} \approx 0$. However, if two or several states are close in energy (see e.g. Figure 5B) or in the neighborhood of a crossing between two (or more) states (see Figure 10B) several weights will differ from 0. This is similar in the multi state adiabatic reactive MD approach where the weights, however, depend on the energy gap between the lowest energy state for a given conformation and all higher excited states. ${ }^{49}$ Because the weights depend on the geometry of the part which is treated with MS-VALBOND, the charge on atom $k$ will also depend on the geometry which effectively amounts to a fluctuating charge

$$
q_{k}(\boldsymbol{r})=\sum_{i=1}^{n_{\mathrm{states}}} w_{i}(\boldsymbol{r}) q_{k}^{i}
$$

where $q_{k}^{i}$ is the charge on atom $k$ in state $i$ and the total charge of the solute must be constant $\sum_{k=1}^{N_{\text {atom }}} q_{k}(\mathbf{r})=q_{\text {solute }}$ for each state (I). The weight $w_{i}=\left(\nu_{0}^{(\mathrm{i})}\right)^{2}$ is the population of resonance structure $I$ which is determined from eigenvector elements $\nu_{0}^{(\mathrm{i})}$ by diagonalizing the $N \times N$ matrix as in equation 1 . Since the weights $w_{i}(\mathbf{r})$ depend on the internal coordinates through the eigenvectors $\nu_{0}^{(i)}$, the derivatives of $w(\mathbf{r})$ with respect to all internal degrees of freedom are required. Although this would, in principle, be possible to be done analytically, it is computationally expensive and cumbersome. Therefore, the derivatives $\frac{\partial w_{i}(\mathbf{r})}{\partial \mathbf{r}}$ are determined 
numerically according to

$$
\begin{aligned}
f_{k, x}= & -\frac{d}{d x}\left(\frac{Q_{\text {solv }} q_{k}(\boldsymbol{r})}{|X-x|}\right) \\
& =-Q_{\text {solv }}\left[\frac{1}{|X-x|} \frac{d q_{k}(\boldsymbol{r})}{d x}+q_{k}(\boldsymbol{r}) \frac{d}{d x}\left(\frac{1}{|X-x|}\right)\right] \\
& =-Q_{\text {solv }}\left[\frac{1}{X} \frac{q_{k} d w_{k}(\boldsymbol{r})}{d x}+q_{k}(\boldsymbol{r}) \frac{d}{d x}\left(\frac{1}{|X-x|}\right)\right] \\
& \approx-Q_{\text {solv }}\left[\frac{q_{k}(x+h, y, z)-q_{k}(x-h, y, z)}{2 h|X-x|}+q_{k}(\boldsymbol{r}) \frac{d}{d x}\left(\frac{1}{|X-x|}\right)\right]
\end{aligned}
$$

where, $X$ and $x$ are the coordinates of the solvent and solute atoms, respectively.

\section{Applications}

MS-VALBOND parametrizations and simulations were performed for the $\mathrm{ClF}_{3}$ molecule, cisplatin and the $\mathrm{BrMe}+\mathrm{Cl}^{-} \rightarrow \mathrm{Br}^{-}+\mathrm{MeCl}$ reaction. $\mathrm{ClF}_{3}$ is a paradigm system for a small hypervalent molecule with an unusual shape. Cisplatin is a hypervalent transition metal complex used as an anti-cancer chemotherapeutic agent ${ }^{63,64}$ and the $\mathrm{S}_{N} 2$ reaction $\mathrm{BrCH}_{3}+\mathrm{Cl}^{-}$ $\rightarrow \mathrm{Br}^{-}+\mathrm{CH}_{3} \mathrm{Cl}$ has been characterized experimentally ${ }^{65,66}$ in the gas- and condensed phase which provides necessary benchmark/validation and it is also one of the standard systems for QM/MM simulations. ${ }^{67}$ These three test systems serve as typical examples to illustrate and test the applicability of MS-VALBOND.

\subsection{Dynamics of $\mathrm{ClF}_{3}$ in Gas Phase and in Solution}

For the parametrization of the diagonal states of $\mathrm{ClF}_{3}$ the core fragment $\mathrm{ClF}_{2}^{+}$was considered. A Morse potential was fitted to reference $a b$ initio data for the Cl-F bond obtained at the CCSD/aug-cc-pVDZ ${ }^{68,69}$ level of theory, computed on a grid ranging from $1.3 \AA$ to 4.0 $\AA$ with a $0.1 \AA$ increment for all six angles $60^{\circ}, 90^{\circ}, 100^{\circ}, 120^{\circ}, 150^{\circ}$ and $180^{\circ}$. The F-Cl-F 
angle $\alpha$ is described by VALBOND and the force constant was fitted to reproduce reference calculations at $60^{\circ}, 90^{\circ}, 100^{\circ}, 120^{\circ}, 150^{\circ}$ and $180^{\circ}$. All fitted force field parameters for the three resonance structures are provided in Table 1. 


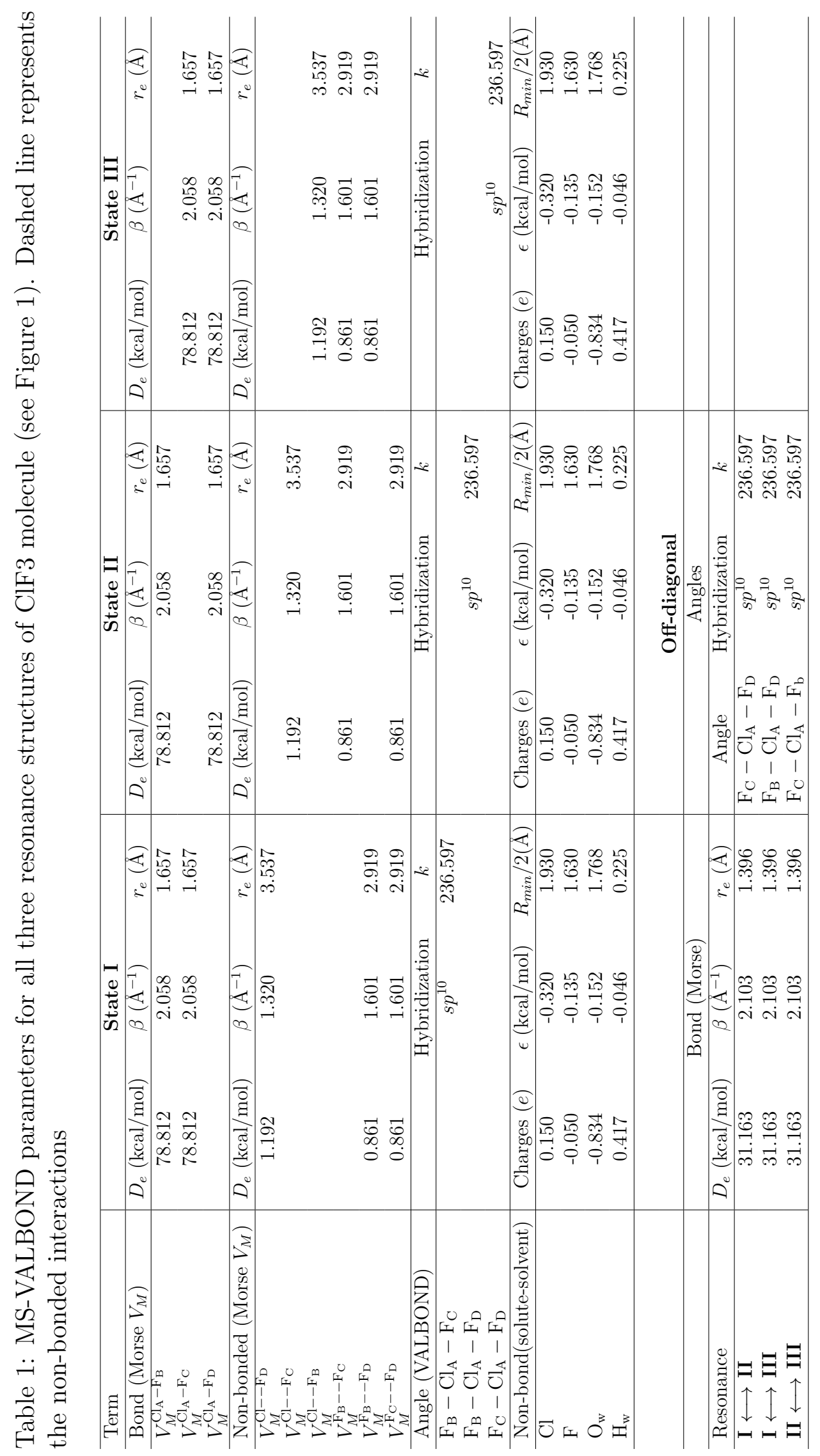


In the second step the parametrization of the off-diagonal terms was carried out by considering six degrees of freedom in $\mathrm{ClF}_{3}$ system: $\mathrm{Cl}_{\mathrm{B}}, \mathrm{Cl}-\mathrm{F}_{\mathrm{C}}, \mathrm{Cl}-\mathrm{F}_{\mathrm{D}}, \mathrm{F}_{\mathrm{B}}-\mathrm{Cl}-\mathrm{F}_{\mathrm{C}}, \mathrm{F}_{\mathrm{C}}-\mathrm{Cl}-\mathrm{F}_{\mathrm{D}}$ and $\mathrm{F}_{\mathrm{B}}-\mathrm{Cl}-\mathrm{F}_{\mathrm{C}}(-) \mathrm{F}_{\mathrm{D}}$ dihedral angle. The off-diagonal force field parameters for $\mathrm{ClF}_{3}$ are also provided in Table 1.

With the FF available, first the system was energy minimized, heated to $300 \mathrm{~K}$ and then equilibrated for 500 ps. Further 500 ps of $N V E$ simulations were performed for the analysis. The solvated system was generated by immersing the solute molecule in a cubic TIP3P water box $^{70}$ of length $30 \AA$.

As a validation, the energy minimized geometry using this parametrization was compared with experimental data ${ }^{71}$ and with the optimized structure obtained at the CCSD/aug-ccpVDZ level of theory (see Table 2). In general, the geometrical parameters for bond lengths and bond angles obtained from MS-VALBOND agree well with both sets of reference data. The $\mathrm{Cl}-\mathrm{F}_{\mathrm{B}, \mathrm{D}}$ bond lengths from MS-VALBOND deviate by $-0.02 \AA$ and $-0.06 \AA$ from the CCSD calculations and experiment, respectively. Similar observations are made for the Cl$\mathrm{F}_{\mathrm{C}}$ bond which differs by $-0.04 \AA$ and $-0.08 \AA$ from the CCSD calculations and experiment. For the $\mathrm{F}_{\mathrm{B}}-\mathrm{Cl}-\mathrm{F}_{\mathrm{D}}$ angle the difference between MS-VALBOND calculations and the reference data is $\sim 2^{\circ}$ (see Table 2) which increases to $6^{\circ}$ for the $\mathrm{F}_{\mathrm{B}}-\mathrm{Cl}-\mathrm{F}_{\mathrm{C}}$ angle. All three approaches yield a planar geometry of the molecule.

Table 2: Comparison of selected geometrical parameters for $\mathrm{ClF}_{3}$ with their corresponding NMR data. ${ }^{71}$ The bond distances are in $\AA$ and angles are in degree.

\begin{tabular}{l|r|r|r}
\hline Geometric parameters & MS-VALBOND & CCSD & NMR structure \\
\hline Cl- $\mathrm{F}_{\mathrm{B}, \mathrm{D}}$ & 1.76 & 1.74 & 1.70 \\
$\mathrm{Cl}-\mathrm{F}_{\mathrm{C}}$ & 1.68 & 1.64 & 1.60 \\
$\mathrm{~F}_{\mathrm{B}}-\mathrm{Cl}-\mathrm{F}_{\mathrm{D}}$ & 172.2 & 173.6 & 174.6 \\
$\mathrm{~F}_{\mathrm{B}}-\mathrm{Cl}-\mathrm{F}_{\mathrm{C}}$ & 93.9 & 86.8 & 87.3 \\
$\mathrm{~F}_{\mathrm{B}}-\mathrm{Cl}-\mathrm{F}_{\mathrm{C}}(-) \mathrm{F}_{\mathrm{D}}$ & 180.0 & 180.0 & 180.0 \\
\hline
\end{tabular}


During the entire $N V E$ simulation the total energy is conserved to within fractions of a $\mathrm{kcal} / \mathrm{mol}$ whether the simulation is carried out in gas phase or in aqueous medium (see Figure 4). For $\mathrm{ClF}_{3}$ in the gas phase, the energy fluctuates within $0.005 \mathrm{kcal} / \mathrm{mol}$ (bottom left in Figure 4) around its average value which increases to $0.2 \mathrm{kcal} / \mathrm{mol}$ in solution (red distribution bottom right in Figure 4). The energy fluctuations $\delta E$ for the same simulation without the solute (i.e. pure water) is comparable, $\sim 0.2 \mathrm{kcal} / \mathrm{mol}$ (blue distribution bottom right Figure 4). It is also of interest to consider the weights of the different resonance structures I to III, see top panels in Figure 4. To describe the correct T-shaped geometry of the $\mathrm{ClF}_{3}$ molecule (see Figure 1), a $s p^{10}$ hybridization was used as had been done in previous work. ${ }^{36}$ Since all the $s p^{10}$ hybrid orbitals have very high $p$-character the equilibrium angle between them is close to $90^{\circ}$. Therefore, resonance structures with an F-Cl-F equilibrium angle close to $90^{\circ}$ (equivalent states I and III) are lower in energy and more highly populated (green and black traces in top row of Figure 4) compared to state II (red trace). Whether the simulation is carried out in gas-phase or in aqueous solution the weights of each of the resonance structures remain similar (see Figure 4) although the fluctuations increase somewhat in water. 


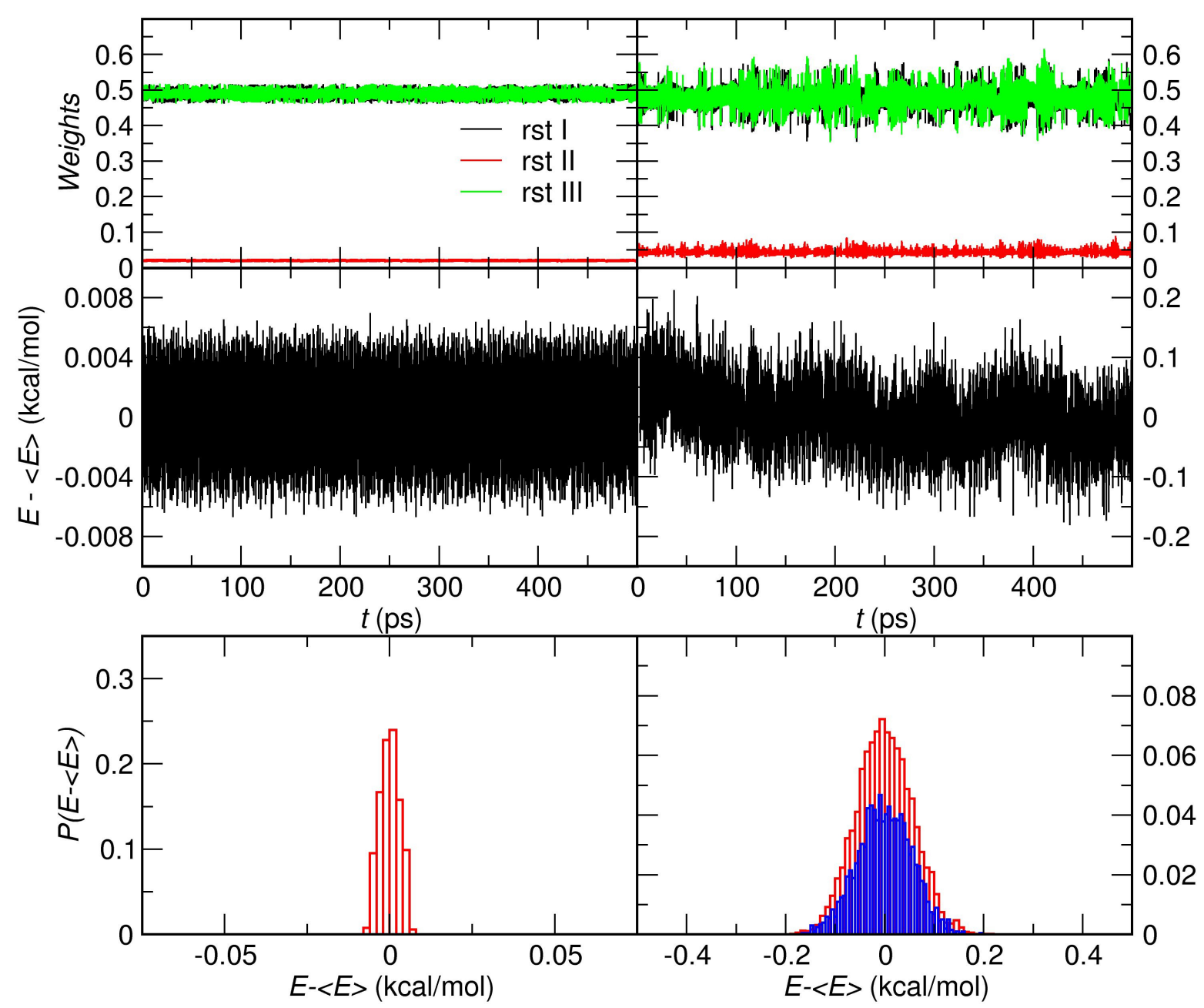

Figure 4: Weights (top), energy fluctuation (middle) and distribution around the mean (bottom) for simulations of $\mathrm{ClF}_{3}$ in the gas phase (left) and in aqueous solution (right). The top panel shows the contribution of each resonance structure I to III (see Figure 1) towards the total structure of the $\mathrm{ClF}_{3}$ molecule during the MD simulations. The energy fluctuations for simulations with (red) and without (blue) the solute in water are shown in the bottom right panel.

Combining all three resonance structures it is possible to describe pseudorotation in $\mathrm{ClF}_{3}$ i.e $\mathrm{F}$ atoms exchange between apical and equatorial positions. For this, an MD simulation was started at the transition state separating the apical and equatorial positions. Figure 5 panel A shows the change of $\mathrm{F}_{\mathrm{B}}-\mathrm{Cl}_{\mathrm{A}}-\mathrm{F}_{\mathrm{C}}$ angle and describes the switching of $\mathrm{F}_{\mathrm{C}}$ position from equatorial to apical. Hence, the MS-VALBOND force field allows to estimate the barrier involved for the transitions among different T-shape arrangements. By considering the 
$\mathrm{F}_{\mathrm{B}}-\mathrm{Cl}_{\mathrm{A}}-\mathrm{F}_{\mathrm{C}}$ angle, free energy for the pseudorotation in $\mathrm{ClF}_{3}$ was computed and found to be $4.3 \mathrm{kcal} / \mathrm{mol}$ (see Figure 5) which agrees very favourably with a value of $4.8 \mathrm{kcal} / \mathrm{mol}$ from NMR experiments ${ }^{71}$ without adjusting the parametrization of the MS-VALBOND force field.

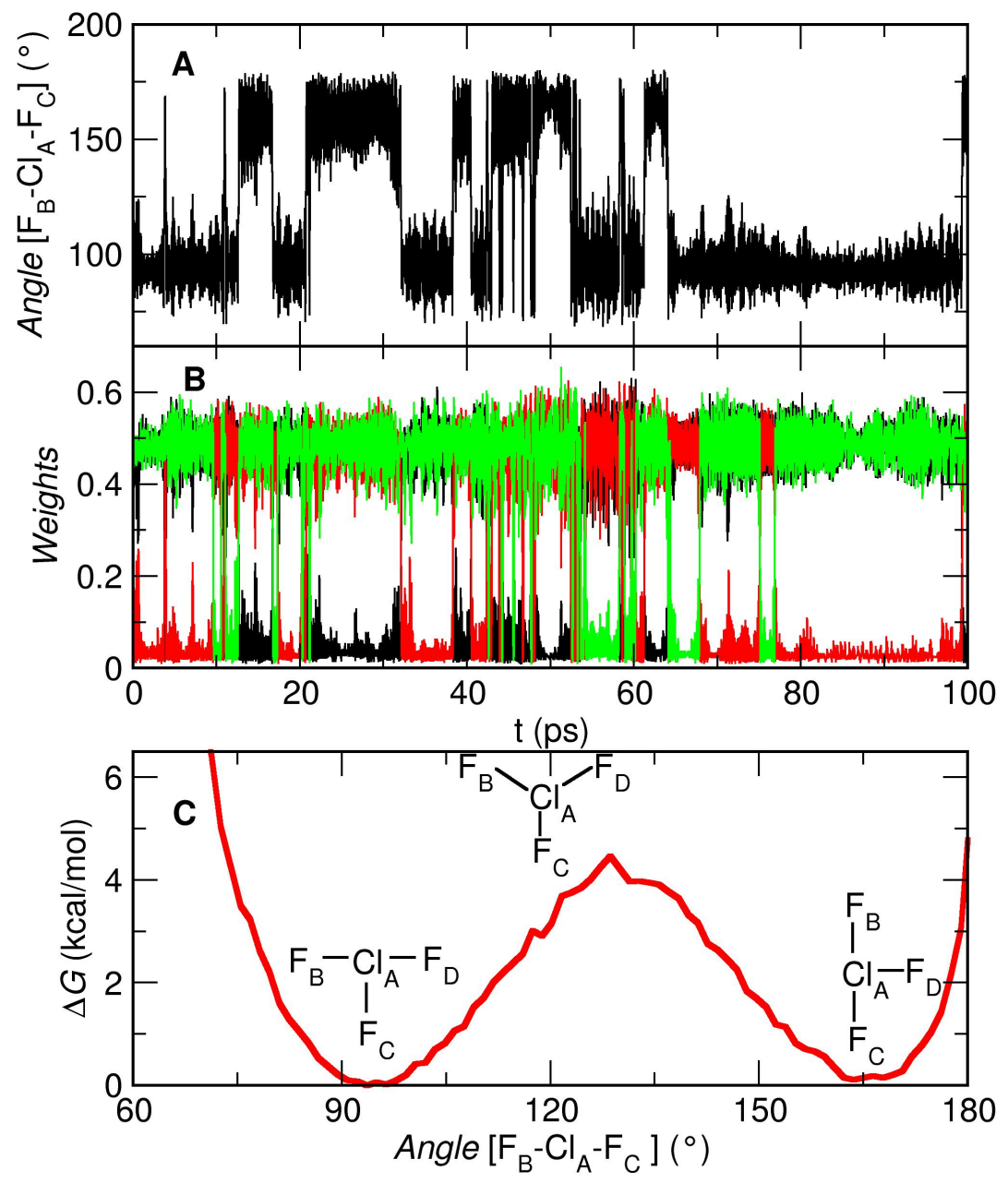

Figure 5: Change of F-Cl-F angle (panel A) and weights (panel B) during the dynamics of $\mathrm{ClF}_{3}$ in the gas phase. The computed free energy profile for the pseudorotation in $\mathrm{ClF}_{3}$ is shown in panel C. Insets in panel $\mathrm{C}$ are the structures representing the two isoenergetic minima and the TS states separating them and involved in the pseudorotation of $\mathrm{ClF}_{3}$. Color code in panel B: resonance structure I (black), II (red) and III (green). 


\section{Structure and Dynamics of Cisplatin}

Cisplatin (cis-diamminedichloridoplatinum(II) (CDDP)) is a Platinum-containing metal complex with two ammonia and two chloride ligands cis to each other. Cisplatin is one of the most important and effective anti-cancer agents used in the treatment of various types of cancers including testicular, ovarian, cervical, or breast cancer. ${ }^{63,64}$ Furthermore, this compound is also used in Auger therapy. ${ }^{72}$ Experimentally, cisplatin was also well characterized spectroscopically which provides a useful basis to validate the present calculations in a meaningful fashion. ${ }^{73}$

The six relevant resonance structures (in cisplatin, $\mathrm{Pt}$ is $\mathrm{sd}^{1}$ hybridized) are shown in Figure 6. For parametrizing the diagonal states, the Pt-Cl and $\mathrm{Pt}-\mathrm{N}\left(\mathrm{H}_{3}\right)$ bonds were fitted to reference data from electronic structure calculations with the B3LYP functional ${ }^{74}$ and the LANL2DZ basis ${ }^{75}$ set for Pt and $6-31 \mathrm{G}(\mathrm{d}, \mathrm{p})^{76}$ for all other atoms, respectively. The necessary reference data were collected by scanning along the $\mathrm{Pt}-\mathrm{Cl}$ bond in $\mathrm{PtCl}_{2}$ and along the Pt-N bond in $\left[\operatorname{Pt}\left(\mathrm{NH}_{3}\right)_{2}\right]^{2+}$ on a grid from $1.5 \AA$ to $5.5 \AA$ with an increment of $0.1 \AA$. To these references data two Morse potentials for Pt-N and Pt-Cl bonds were fitted and parameters are given in the SI (see Table S1).

For the parametrization of the off-diagonal terms, the element $V_{12}$ (transition between states I and II) is considered as an example. In going from resonance structure I to II, one Pt$\mathrm{N}$ bond breaks and one Pt-Cl bond forms. Therefore, the off-diagonal term is described by a sum of two Morse potentials $V_{\mathrm{Pt}-\mathrm{N}}$ and $V_{\mathrm{Pt}-\mathrm{Cl}}$ which are combined according to $[\cos (\theta)-1]^{2} V_{\mathrm{Pt}-\mathrm{N}}+[\cos (\theta)-1]^{2} V_{\mathrm{Pt}-\mathrm{Cl}}$, see Methods. Here, $\theta$ is the N-Pt-Cl angle and the corresponding bending potential is described by VALBOND. Thus, in total six parameters (three for each Morse) need to be fitted to the reference data. For that, five degrees of freedom were considered; the Pt-Cl and Pt-N $\left(\mathrm{H}_{3}\right)$ distances, and the Cl-Pt-Cl, Cl-Pt-N $\left(\mathrm{H}_{3}\right)$ and $\left(\mathrm{H}_{3}\right) \mathrm{N}-\mathrm{Pt}-\mathrm{N}\left(\mathrm{H}_{3}\right)$ angles for the parametrization of the off-diagonal elements. A total 
of 5821 reference energies and the reference structures were generated by considering five degrees of freedom; the Pt-Cl and Pt- $\mathrm{N}\left(\mathrm{H}_{3}\right)$ distances $(1.5 \AA$ to $3.9 \AA$ with 0.2 increment), and the Cl-Pt-Cl, Cl-Pt-N $\left(\mathrm{H}_{3}\right)$ and $\left(\mathrm{H}_{3}\right) \mathrm{N}-\mathrm{Pt}-\mathrm{N}\left(\mathrm{H}_{3}\right)$ angles (ranging from $60^{\circ}$ to $120^{\circ}$ with $\Delta \theta=10^{\circ}$ ). The off-diagonal parameters were fitted to reference $a b$ initio data by non-linear least square fitting using NL2SOL algorithm. ${ }^{77}$

The partial charges on each atom were those from a Natural Bond Orbital (NBO) ${ }^{78}$ analysis of the $\left[\mathrm{Pt}\left(\mathrm{NH}_{3}\right)_{2} \mathrm{Cl}_{2}\right]$ complex in its energy minimized structure using the B3LYP functional and the LANL2DZ basis set for Pt and 6-31G(d,p) for all other atoms. Van der Waals interactions within the solute were again modeled using Morse potentials and are parametrized with respect to interaction between each atom and He as a probe (see above). If the atoms are bonded to each other or to a common atom then they are considered as bonded. For example, in resonance structure I (see Figure 6), two $\mathrm{Cl}$ atoms are unbound and all the nonbonded interactions with $\mathrm{Pt}, \mathrm{N}$ and $\mathrm{Cl}$ atoms were computed. The Morse parameters for each atom were obtained by fitting the Morse potential with the potential energy curve obtained along the $\mathrm{Pt} / \mathrm{Cl} / \mathrm{N}-\mathrm{He}$ bond, see Methods. The remaining parameters for the $\mathrm{NH}_{3}$ ligand are those from the CGenFF force field ${ }^{79}$ and a summary of all parameters is provided in Table 3.

Table 3: Model systems from which reference electronic structure data was determined for parametrization of diagonal and off-diagonal terms.

\begin{tabular}{c|c|c} 
Diagonal Term & Model & Potential \\
\hline Pt-Cl & $\mathrm{PtCl}_{2}$ & Morse \\
Pt-N & {$\left[\mathrm{Pt}\left(\mathrm{NH}_{3}\right)_{2}\right]^{2+}$} & Morse \\
Angle (L-M-L) & {$\left[\mathrm{Pt}\left(\mathrm{NH}_{3}\right)_{2} \mathrm{Cl}_{2}\right]$} & VALBOND \\
Non-bonded & $\mathrm{Pt} / \mathrm{N} / \mathrm{Cl} / \mathrm{H}-\mathrm{He}$ & Morse \\
Off diagonal Term & {$\left[\mathrm{Pt}\left(\mathrm{NH}_{3}\right)_{2} \mathrm{Cl}_{2}\right]$} & Scaled Morse (see equation 11) \\
\hline
\end{tabular}




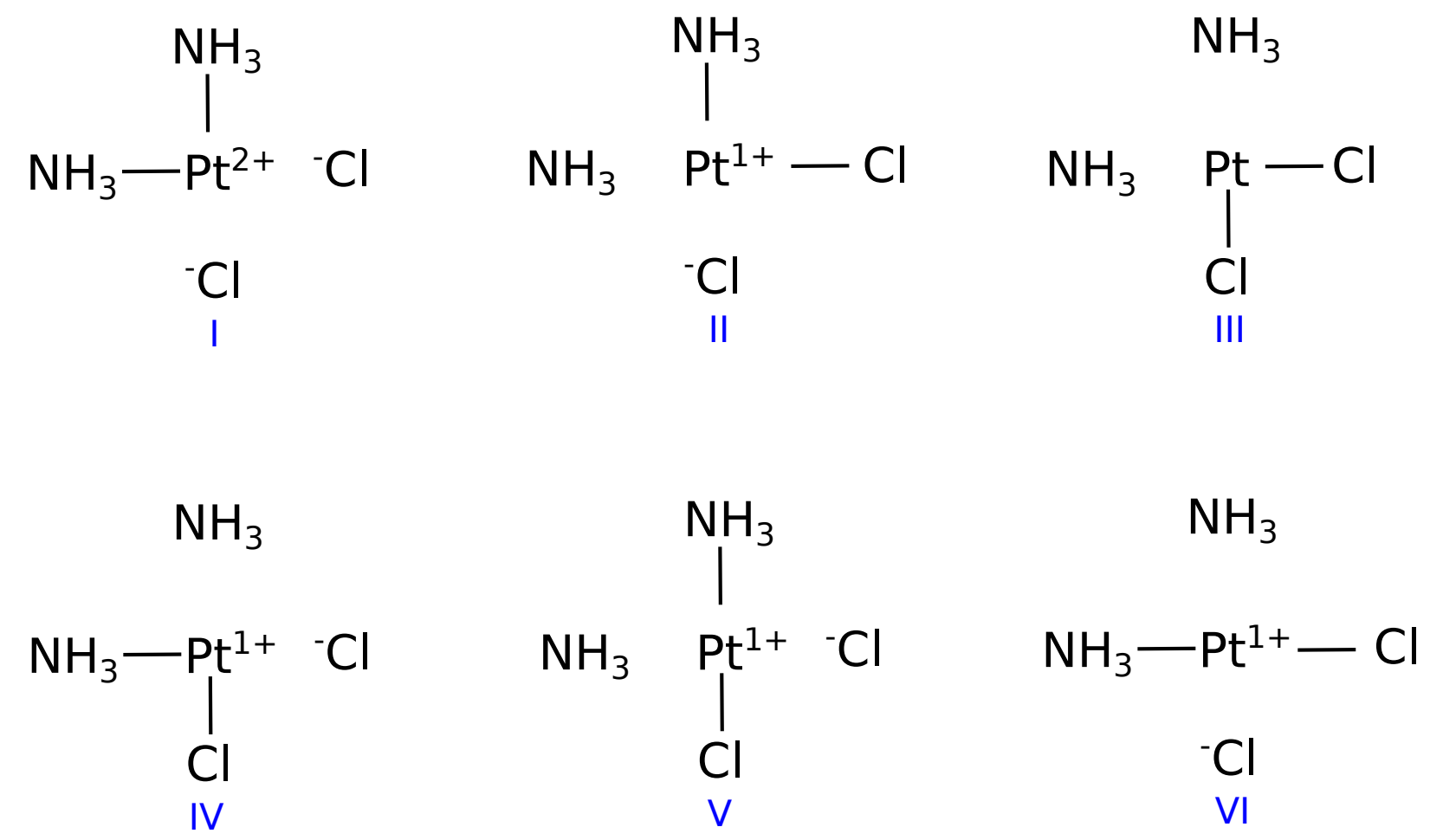

Figure 6: The six resonance structures of cisplatin. Resonance structures II and IV are chemically equivalent to structures V and VI. NBO analysis with Natural Resonance Theory ${ }^{39}$ demonstrates that all six resonance structures exhibit significant population depending on the molecular geometry.

For structural validation, the minimized geometry from MS-VALBOND was compared with the corresponding crystal structure, ${ }^{73}$ see Figure 7 and Table 4 . It is found that bond lengths and angles from MS-VALBOND agree to within less than $2 \%$ with the X-ray data. Specifically, MS-VALBOND yields bond distances Pt-N and Pt-Cl in cisplatin of $2.07 \AA$ and 2.36 $\AA$, respectively, compared with $2.05 \AA$ and $2.32 \AA$ from the X-ray crystal structure. ${ }^{73}$ Also, the deviation for all three angles (N-Pt-N, Cl-Pt-Cl and Cl-Pt-N) compared to their experimental ${ }^{73}$ values are less than $2^{\circ}$. 


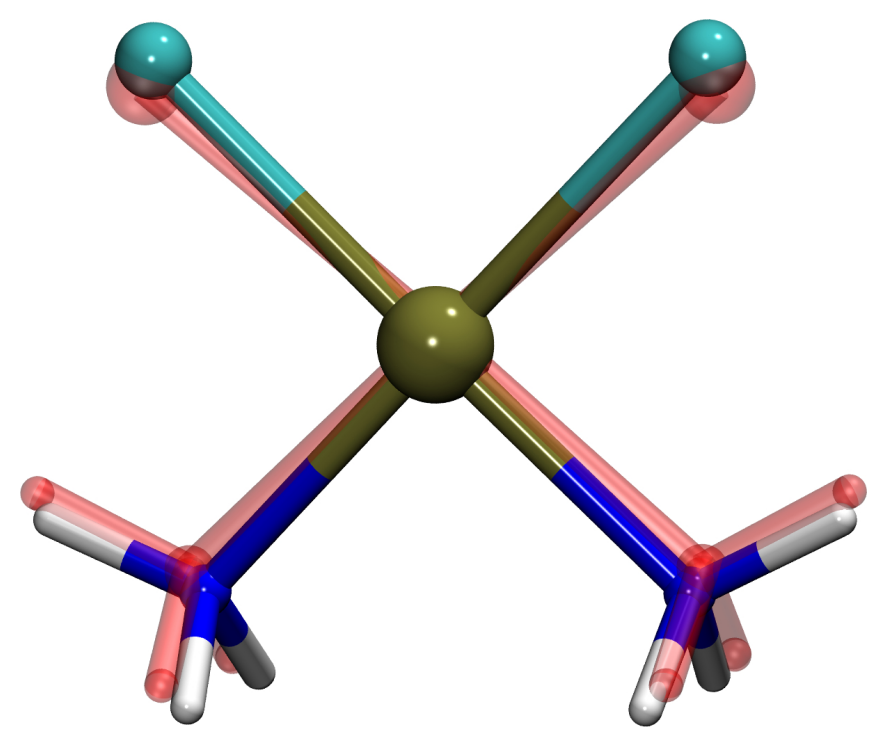

Figure 7: MS-VALBOND optimized structures of cisplatin along with the crystal structure ${ }^{73}$ (transparent red). Atom color code used, Pt (ocher), Cl (cyan), N (blue) and H (white).

Table 4: Comparison of selected geometrical parameters with their corresponding crystal data. ${ }^{73}$ The bond distances are in $\AA$ and angles are in degree.

\begin{tabular}{c|c|c}
\hline Geometric parameters & MS-VALBOND & Experiment $^{73}$ \\
\hline Pt-Cl & 2.36 & 2.32 \\
Pt-N & 2.07 & 2.05 \\
Cl-Pt-Cl & 90.1 & 91.6 \\
N-Pt-N & 91.7 & 90.2 \\
Cl-Pt-N & 87.6 & 88.9 \\
\hline
\end{tabular}

A further validation of the MS-VALBOND force field for cisplatin was done by computing the infrared spectrum without adjusting the parametrization. The spectrum was calculated from the dipole moment auto-correlation function $C(t)=<\mu(0) \mu(t)>$ from a 1 ns MD simulation in gas phase. The total dipole moment $\mu(t)$ was recorded along the trajectory and correlated over $2^{15}$ time origins separated by 1 fs to yield $C(t)$. Then the spectral signal $C(\omega)$ was computed from the fast Fourier transform (FFT) with a Blackman filter. Finally, the infrared spectrum $A(\omega)$ was determined by Boltzmann weighting $C(\omega)$ according to

$$
A(\omega)=\omega\left[1-\exp \left(-\frac{\omega}{k_{B} T}\right)\right] C(\omega)
$$


Here $\omega, k_{B}$ and $T$ are the frequency, Boltzmann constant and temperature, respectively. Figure S2 reports the computed IR-spectra (black trace) compared with the experimentally determined $^{80}$ vibrational frequencies (no intensity information is available from experiments). A direct comparison for the most important modes is provided in Table 5 and shows that they compare favourably. This is particularly true for the L-M-L bending frequencies for all three N-Pt-N, Cl-Pt-Cl and N-Pt-Cl angles which match very well with their experimental frequencies $^{80}$ (see Table 5). On the other hand the Pt-N and Pt-Cl stretch frequency deviate by about $50 \mathrm{~cm}^{-1}$ from the experiments. ${ }^{80}$ Finally, the peak at $\sim 900 \mathrm{~cm}^{-1}$ in the computed spectrum corresponds to the $\mathrm{NH}_{3}$ wagging vibration and also agrees closely with experiment (at $927 \mathrm{~cm}^{-1}$ ). Further improvement could be achieved by explicitly fitting for example the stretching force constants to adjust the Pt-N, Pt-Cl or N-H stretch frequencies which was, however, not pursued here.

Table 5: Calculated IR spectrum from the dipole moment auto-correlation function form MD simulations of $1 \mathrm{~ns}$ and experimental vibrational frequencies of cisplatin. Assignments are based on the power spectra for each bond distances and angles mentioned.

\begin{tabular}{l|c|c}
\hline Assignment & Computed Frequency $\left(\mathrm{cm}^{-1}\right)$ & Experimental Frequency ${ }^{80}\left(\mathrm{~cm}^{-1}\right)$ \\
\hline$\nu_{\mathrm{Pt}-\mathrm{N}}$ & 410 & 469 \\
$\nu_{\mathrm{Pt}-\mathrm{Cl}}$ & 360 & 323 \\
$\nu_{\mathrm{N}-\mathrm{Pt}-\mathrm{N}}$ & 258 & 256 \\
$\nu_{\mathrm{Cl}-\mathrm{Pt}-\mathrm{Cl}}$ & 159 & 156 \\
$\nu_{\mathrm{Cl}-\mathrm{Pt}-\mathrm{N}}$ & 75 & $92-99$ \\
$\delta_{\mathrm{NH}}$ (wag) & 881 & 927 \\
$\delta_{\mathrm{NH}}$ (bend) & 1510 & 1545 \\
$\nu_{\mathrm{N}-\mathrm{H}}$ & 3415 & 3500 \\
\hline
\end{tabular}

Finally, the quality of the MS-VALBOND force field was also established by comparing to electronic structure calculations for thermally populated structures. For this an MD simulation at $50 \mathrm{~K}$ was carried out from which 50 structures were randomly selected. Their energies were determined at the B3LYP level of theory with the 6-31G(d,p) basis set for all atoms except Pt for which LANL2DZ basis is used. These energies are then correlated with the 
MS-VALBOND and shown in Figure 8. The root mean square deviation in energy $\left(\mathrm{RMSD}_{\mathrm{E}}\right)$ between DFT and MS-VALBOND is $0.33 \mathrm{kcal} / \mathrm{mol}$ which is within chemical accuracy and further demonstrates the quality of the MS-VALBOND parametrization for this TM complex.

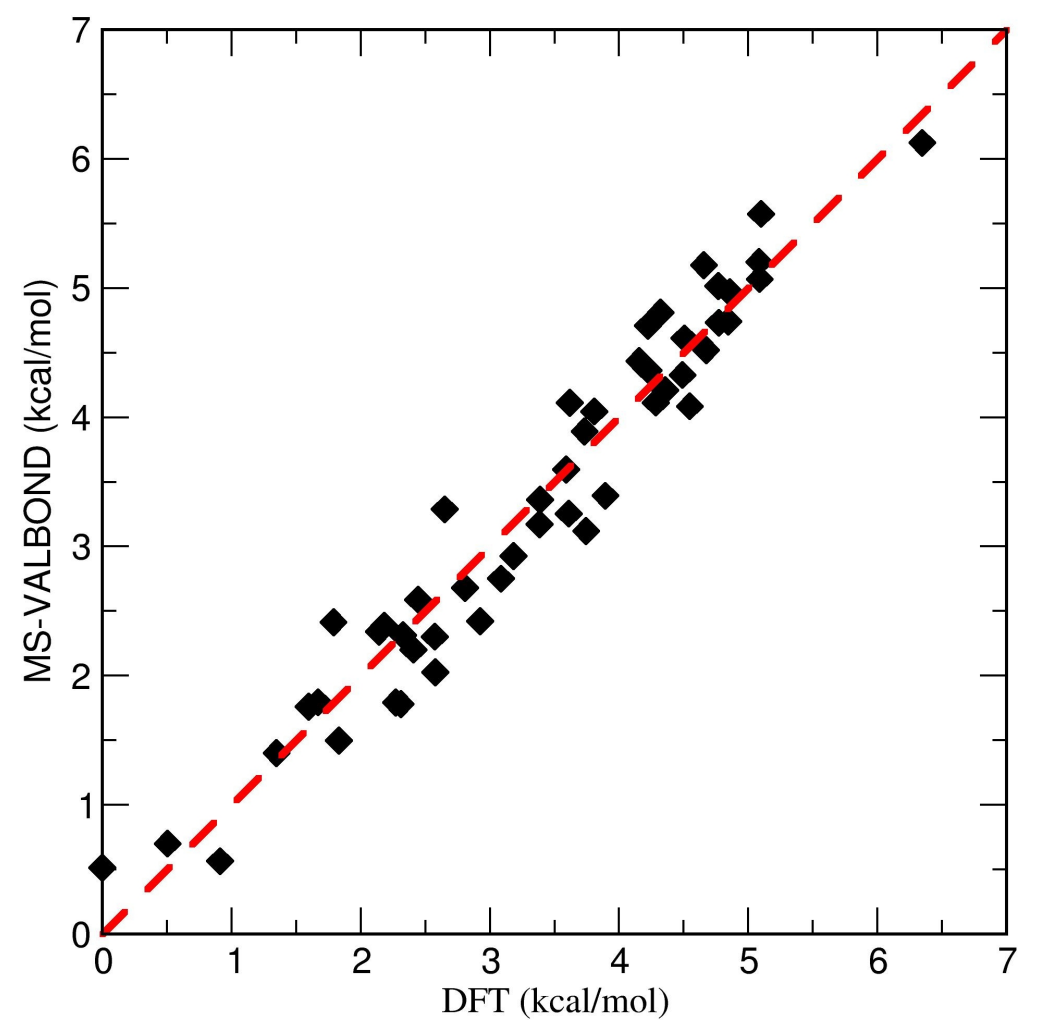

Figure 8: Energy comparison of 50 structure obtained from MD simulations MS-VALBOND and DFT (B3LYP/6-31G(d,p) and LANL2DZ basis on Pt). The RMSDE between two methods is $0.33 \mathrm{kcal} / \mathrm{mol}$.

Figure 9 shows the variation in occupation number of each resonant structure of cisplatin during the MD simulations. From Figure 9, it is clear that resonant structure I has the largest occupation number because the two bonded ligands $\left(\mathrm{NH}_{3}\right)$ are strong ligands and the angle between them is $90^{\circ}$ which is the equilibrium angle for $\mathrm{sd}^{1}$ hybridization. It is also noticed that because the two $\mathrm{Cl}^{-}$are weaker ligands, state III has a lower occupation number compared to state I even though the angle between the ligands is $90^{\circ}$. Both resonant structures II and IV are chemically equivalent and have similar occupation number distribution 
(see top panel Figure 9). Since the angle between the bound ligands for resonant structure $\mathrm{V}$ and VI is $180^{\circ}$ and is far from the $\mathrm{sd}^{1}$ hybridization angle $\left(90^{\circ}\right)$, they are highest in energy and their occupation is lowest.
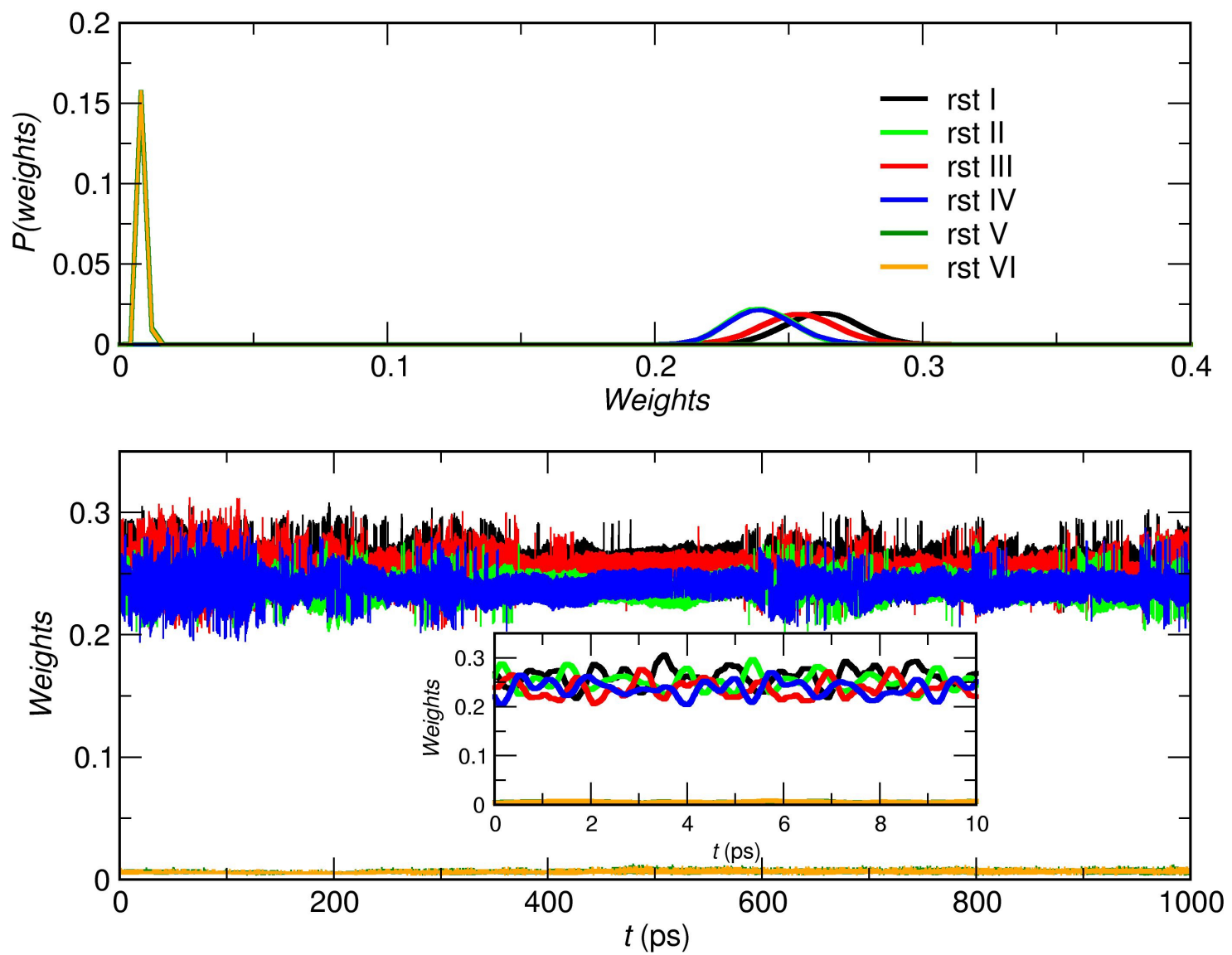

Figure 9: Variation of occupation number (bottom) of each resonance structure of cisplatin during the MD simulations and corresponding probability distribution (top) are shown here.

\section{The $\mathrm{BrMe}+\mathrm{Cl}^{-}$Reaction}

As a last example the bimolecular nucleophilic substitution $\left(\mathrm{S}_{N} 2\right)$ reaction between methylbromide and chloride ion in aqueous solution is studied. Bromomethane $\left(\mathrm{CH}_{3} \mathrm{Br}\right)$ is used as fumigant for several pests like mites, fungi, spiders etc. ${ }^{81}$ Methylbromides are mainly used for soil fumigation. However, their exposure to the environment are very hazardous as they are 
responsible for the ozone depletion in stratosphere. ${ }^{81,82}$ On the other hand chlorine species are found in marine boundary layer where $\mathrm{CH}_{3} \mathrm{Br}$ enters into the atmosphere. Thus, they can help in destructing hazardous methylbromides by reacting with them. $\mathrm{S}_{N} 2$ reactions and the solvent effects on the kinetics are very important in organic chemistry. ${ }^{83}$ Also, the $\mathrm{BrMe}+\mathrm{Cl}^{-} \rightarrow \mathrm{Br}^{-}+\mathrm{MeCl} \mathrm{S} N_{2}$ reaction has been studied both theoretically ${ }^{66,83,84}$ and experimentally. ${ }^{65,85}$

At the MP2/6-311++G(d,p) and MP2/aug-cc-pVTZ level of theory the enthalpic barrier for the $\mathrm{BrMe}+\mathrm{Cl}^{-} \rightarrow \mathrm{Br}^{-}+\mathrm{MeCl}$ reaction in implicit water (polarizable continuum model $(\mathrm{PCM}), \epsilon=78.5)$ is 24.6 and $22.4 \mathrm{kcal} / \mathrm{mol},{ }^{66}$ respectively. This compares with the experimentally measured free energy of activation of $24.7 \mathrm{kcal} / \mathrm{mol} .{ }^{65,85}$ Previous calculations for halomethane substitution reactions at the MP2 and CCSD level of theory in implicit water also yielded favourable agreement with experiment whereas DFT underestimated the barrier height. ${ }^{66,86}$ As a comparison, the gas-phase barrier for the $\mathrm{BrMe}+\mathrm{Cl}^{-} \rightarrow \mathrm{Br}^{-}+\mathrm{MeCl} \mathrm{reac}^{-}$ tion from CCSD/aug-cc-pVDZ calculations is found to be $11.7 \mathrm{kcal} / \mathrm{mol}$, in close agreement with previously computed $11.2 \mathrm{kcal} / \mathrm{mol}$ and experimental values $9.5 \mathrm{kcal} / \mathrm{mol}{ }^{87,88}$ The free energy profile for the gas phase reaction is reported in Figure S3.

For the MS-VALBOND parametrization of the $\mathrm{BrMe}+\mathrm{Cl}^{-} \rightarrow \mathrm{Br}^{-}+\mathrm{MeCl} \mathrm{S}_{\mathrm{N}} 2$ reaction, the diagonal states were described by two Morse potentials and fitted to reference data from CCSD/aug-cc-pVDZ calculations along the C-Br and C-Cl bonds in BrMe or MeCl, respectively. All the $\mathrm{Cl} / \mathrm{Br}-\mathrm{C}-\mathrm{H}$ angles were described by VALBOND with a $s p^{3}$ hybridization. For the off-diagonal states, the parametrization was carried out by considering two degrees of freedom $\mathrm{C}-\mathrm{Br}$ and $\mathrm{C}-\mathrm{Cl}$ bonds in $[\mathrm{Br}-\mathrm{Me}-\mathrm{Cl}]^{-}$and fitted to Morse potentials $V_{\mathrm{Me}-\mathrm{Br}}$ and $V_{\mathrm{Me}-\mathrm{Cl}}$.

To study the $\mathrm{BrMe}+\mathrm{Cl}^{-} \rightarrow \mathrm{Br}^{-}+\mathrm{MeCl}$ reaction in aqueous solution, two reactants $\mathrm{Cl}^{-}$and 
MeBr were solvated in a cubic TIP3P water box of length $30 \AA$. The system is energy minimized, followed by heating and an equilibration of $1 \mathrm{~ns}$ at $300 \mathrm{~K}$. The energy barrier of 24.7 $\mathrm{kcal} / \mathrm{mol}$ (see above) associated with the substitution reaction $\mathrm{BrMe}+\mathrm{Cl}^{-} \rightarrow \mathrm{Br}^{-}+\mathrm{MeCl}$ is too high for directly sampling it in unbiased simulations on time scales accessible to conventional MD simulations. In order to validate the present implementation for following chemical reactions without using a bias the reaction barrier is first artificially reduced to 2.3 $\mathrm{kcal} / \mathrm{mol}$ by decreasing the dissociation energy of the $\mathrm{C}-\mathrm{Br}$ bond. With this modification the reaction occurs on the 10 ps time scale (see Figure 10) in unbiased MD simulations. The total energy is conserved to within $0.2 \mathrm{kcal} / \mathrm{mol}$ (see Figure 10) which confirms that the present implementation is also suitable to follow chemical reactions in a meaningful fashion. 

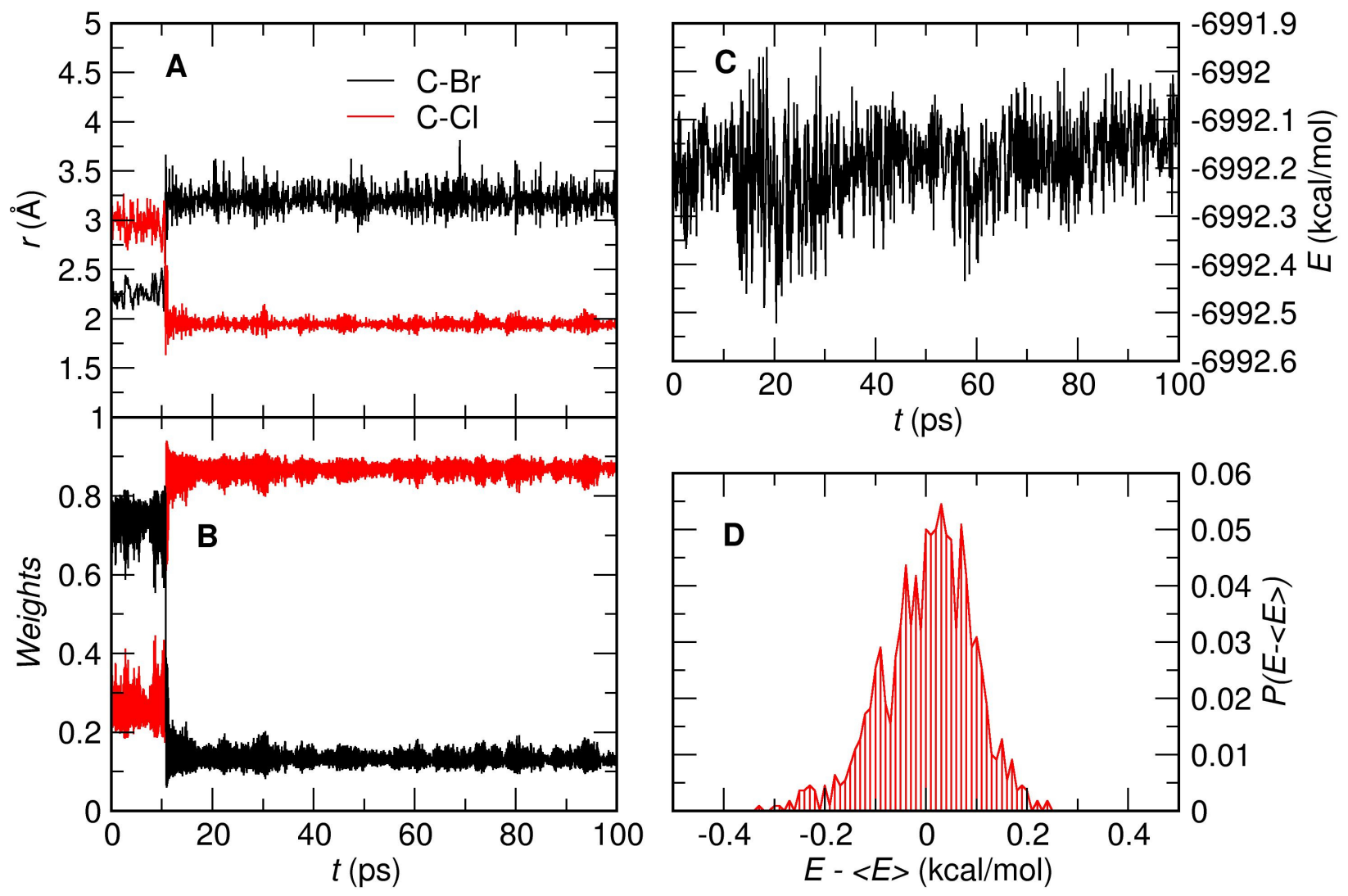

Figure 10: Energy conservation and population of the states for $\mathrm{Me}-\mathrm{Br}+\mathrm{Cl}^{-}$reaction in water with reduced barrier height of $2.3 \mathrm{kcal} / \mathrm{mol}$. Panel A shows the two $\mathrm{C}-\mathrm{Br}$ and $\mathrm{C}-\mathrm{Cl}$ distance traces. Variation of weights for two states reactant and product during the reaction are shown in panel B. Panel C and D are for the change of total energy during the reaction and the corresponding energy fluctuation distribution respectively.

Next, the $\mathrm{S}_{\mathrm{N}} 2$ reaction for $\mathrm{BrMe}$ and $\mathrm{Cl}^{-}$in aqueous solution was investigated using the true potential energy surface with a barrier height of $24.6 \mathrm{kcal} / \mathrm{mol}$. For this, umbrella sampling ${ }^{89}$ simulations were performed. The distance difference $\delta=d_{\mathrm{C}-\mathrm{Br}}-d_{\mathrm{C}-\mathrm{Cl}}$ was used as the reaction coordinate. The system was prepared as described above, followed by a total of 50 umbrella simulations along the reaction coordinate with $-2.5 \leq \delta \leq 2.5$. For each umbrella window 250 ps of equilibrium simulations (100 ps equilibrium simulation and 150 ps for data accumulation) were carried out. For all umbrella windows a force constant $k=50 \mathrm{kcal} / \mathrm{mol}$ was used. 
The weighted histogram analysis method (WHAM) ${ }^{90}$ was used to determine the resultant free energy profile for the $\mathrm{BrMe}+\mathrm{Cl}^{-} \rightarrow \mathrm{Br}^{-}+\mathrm{MeCl}$ reaction in water, see Figure 11. The computed free energy barrier is $24.6 \mathrm{kcal} / \mathrm{mol}$ which agrees well with the experimen$\operatorname{tal}^{65,85}$ value of $24.7 \mathrm{kcal} / \mathrm{mol}$. Also, the barrier for the reverse reaction is $26.7 \mathrm{kcal} / \mathrm{mol}$ which agrees quite well with experiment that finds $27.8 \mathrm{kcal} / \mathrm{mol} .{ }^{65,66}$ Finally, the computed free energy difference $\Delta \Delta G=2.1 \mathrm{kcal} / \mathrm{mol}$ between reactant and product is also consistent with experiment which found $3.1 \mathrm{kcal} / \mathrm{mol}$ (see Figure 11 red line) ${ }^{65,66}$ Probably, further improvement of the force field would be possible by slightly tuning force field parameters such as the $\mathrm{C}-\mathrm{H}$ bonds and $\mathrm{H}-\mathrm{C}-\mathrm{H}$ angles.

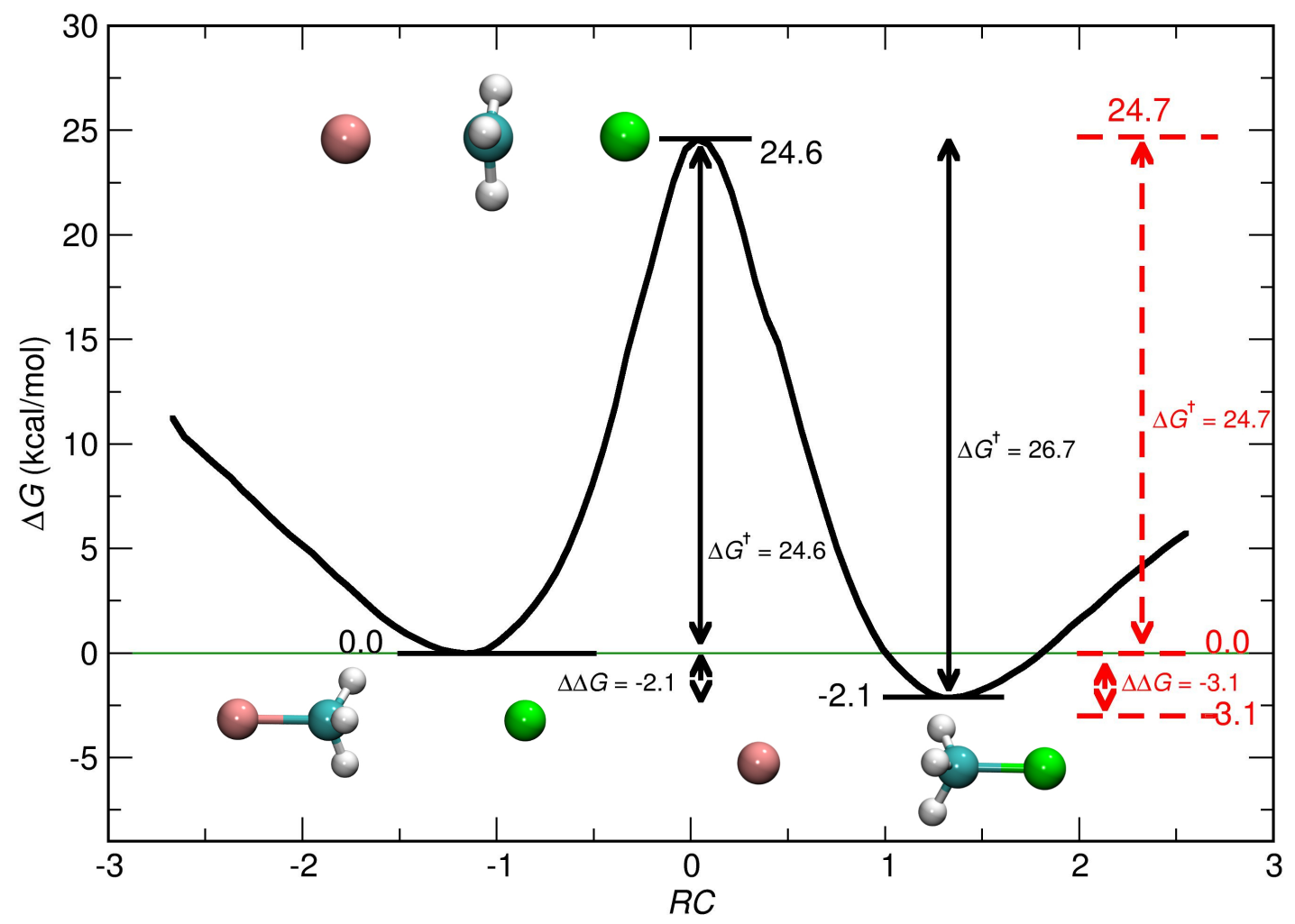

Figure 11: The free energy profile for the $\mathrm{BrMe}+\mathrm{Cl}^{-} \rightarrow \mathrm{Br}^{-}+\mathrm{MeCl}$ reaction in water obtained from the umbrella sampling simulations. Experimental values shown in red. ${ }^{65}$ Atom color code used: $\mathrm{C}$ (cyan), $\mathrm{Br}$ (pink), $\mathrm{Cl}$ (green) and $\mathrm{H}$ (white). For the free energy profile in gas phase see Figure S3. 


\section{Conclusion}

The multi-state VALBOND (MS-VALBOND) approach discussed in the present work has been successfully implemented and applied to a range of situations including hypervalent molecules, transition metal complexes and chemical reactions. It is demonstrated that total energy is conserved and, whenever possible, comparison with experiment leads to good agreement for structures, vibrational frequencies or free energy barriers. Hence, quantitative atomistic simulations for hypervalent and metal-containing systems on extended time scales become possible with the present implementation which allows to make contact with experiments and provide additional molecular-level insight from the analysis of the simulations as is possible from MS-ARMD studies. ${ }^{23,91}$ This is one of the main differences compared with methods such as ReaxFF which are more suitable for qualitative investigations.

The current implementation is also suitable for applications to larger systems, such as proteins because the reactive subsystem (e.g. a protein active site) can be represented with MS-VALBOND whereas the remainder of the simulation is treated with an empirical force field. Given the flexible implementation it is expected that MS-VALBOND can be applied to a range of challenging problems, including transition metals in biological systems or reactions involving organometallic systems.

\section{Acknowledgments}

This work was supported by the University of Basel, and the Swiss National Science Foundation through grants 200021-7117810 and the NCCR MUST (to MM). CRL acknowledges support from the Regents of the University of Wisconsin-Madison. 


\section{References}

(1) Lifson, S.; Warshel, A. Consistent Force Field for Calculations of Conformations Vibrational Spectra and Enthalpies of Cycloalkane and n-Alkane Molecules. J. Chem. Phys. 1968, 49, 5116-5129.

(2) Levitt, M.; Lifson, S. Refinement of Protein Conformations Using a Macromolecular Energy Minimization Procedure. J. Mol. Biol. 1969, 46, 269-279.

(3) Hwang, M. J.; Stockfisch, T. P.; Hagler, A. T. Derivation of Class II Force Fields: 2. Derivation and Characterization of a Class II Force Field, CFF93, for the Alkyl Functional Group and Alkane Molecules. J. Am. Chem. Soc. 1994, 116, 2515-2525.

(4) Maple, J. R.; Hwang, M. J.; Stockfisch, T. P.; Dinur, U.; Waldman, M.; Ewig, C. S.; Hagler, A. T. Derivation of Class-II Force-Fields .1. Methodology and Quantum ForceField for the Alkyl Functional-Group and Alkane Molecules. J. Comput. Chem. 1994, $15,162-182$.

(5) MacKerell, A.; Bashford, D.; Bellott, M.; Dunbrack, R.; Evanseck, J.; Field, M.; Fischer, S.; Gao, J.; Guo, H.; Ha, S. et al. All-atom empirical potential for molecular modeling and dynamics studies of proteins. J. Phys. Chem. B 1998, 102, 3586-3616.

(6) Weiner, S.; Kollman, P.; Case, D.; Singh, U.; Ghio, C.; Alagona, G.; Profeta, S.; Weiner, P. A New Force-Field for Molecular Mechanical Simulation of Nucleic-Acids and Proteins. J. Am. Chem. Soc. 1984, 106, 765-784.

(7) Jorgensen, W.; Tiradorives, J. The OPLS Potential Functions for Proteins - Energy Minimizations for Crystals of Cyclic-Peptides and Crambin. J. Am. Chem. Soc. 1988, 110, 1657-1666.

(8) Stone, A. J. Distributed Multipole Analysis, or how to Describe a Molecular ChargeDistribution. Chem. Phys. Lett. 1981, 83, 233-239. 
(9) Piquemal, J.-P.; Gresh, N.; Giessner-Prettre, C. Improved formulas for the calculation of the electrostatic contribution to the intermolecular interaction energy from multipolar expansion of the electronic distribution. J. Phys. Chem. A 2003, 107, 10353-10359.

(10) Plattner, N.; Meuwly, M. The role of higher CO-multipole moments in understanding the dynamics of photodissociated carbonmonoxide in myoglobin. Biophys. J. 2008, 94, $2505-2515$.

(11) Jorgensen, W. L.; Schyman, P. Treatment of Halogen Bonding in the OPLS-AA Force Field: Application to Potent Anti-HIV Agents. J. Chem. Theory Comput. 2012, 8, $3895-3901$.

(12) Kramer, C.; Gedeck, P.; Meuwly, M. Multipole-Based Force Fields from ab Initio Interaction Energies and the Need for Jointly Refitting All Intermolecular Parameters. J. Chem. Theory Comput. 2013, 9, 1499-1511.

(13) Lee, M. W.; Carr, J. K.; Goellner, M.; Hamm, P.; Meuwly, M. 2D IR spectra of cyanide in water investigated by molecular dynamics simulations. J. Chem. Phys. 2013, 139.

(14) Devereux, M.; Raghunathan, S.; Fedorov, D. G.; Meuwly, M. A Novel, Computationally Efficient Multipolar Model Employing Distributed Charges for Molecular Dynamics Simulations. J. Chem. Theory Comput. 2014, 10, 4229-4241.

(15) Unke, O. T.; Devereux, M.; Meuwly, M. Minimal distributed charges: Multipolar quality at the cost of point charge electrostatics. J. Chem. Phys. 2017, $14 \%$.

(16) Rick, S.; Stuart, S. Potentials and Algorithms for Incorporating Polarizability in Computer Simulations; Reviews in Computational Chemistry; 2002; Vol. 18; pp 89-146.

(17) Shi, Y.; Xia, Z.; Zhang, J.; Best, R.; Wu, C.; Ponder, J. W.; Ren, P. Polarizable Atomic Multipole-Based AMOEBA Force Field for Proteins. J. Chem. Theory Comput. 2013, 9, 4046-4063. 
(18) Lopes, P. E. M.; Huang, J.; Shim, J.; Luo, Y.; Li, H.; Roux, B.; MacKerell, A. D., Jr. Polarizable Force Field for Peptides and Proteins Based on the Classical Drude Oscillator. J. Chem. Theory Comput. 2013, 9, 5430-5449.

(19) Warshel, A.; Levitt, M. Theoretical studies of enzymic reactions: Dielectric, electrostatic and steric stabilization of the carbonium ion in the reaction of lysozyme. J. Mol. Biol. 1976, 103, 227-249.

(20) Alagona, G.; Ghio, C.; Kollman, P. A. Simple-Model for the Effect of Glu165-Asp165 Mutation on the Rate of Catalysis in Triose Phosphate Isomerase. J. Mol. Biol. 1986, $191,23-27$.

(21) Bash, P. A.; Field, M. J.; Karplus, M. Free-Energy Perturbation Method for ChemicalReactions in the Condensed Phase - a Dynamical-Approach Based on a Combined Quantum and Molecular Mechanics Potential. J. Am. Chem. Soc. 1987, 109, 80928094.

(22) Capano, G.; Chergui, M.; Rothlisberger, U.; Tavernelli, I.; Penfold, T. J. A Quantum Dynamics Study of the Ultrafast Relaxation in a Prototypical Cu (I)-Phenanthroline. J. Phys. Chem. A 2014, 118, 9861-9869.

(23) Das, A. K.; Meuwly, M. Kinetics and Structural Interpretation of Competitive Ligand Binding for NO Dioxygenation in Truncated Hemoglobin N. Angew. Chem. Int. Ed. 2018, in print, .

(24) Gaus, M.; Cui, Q.; Elstner, M. DFTB-3 ${ }^{r d}$ : Extension of the Self-Consistent-Charge Density-Functional Tight-Binding Method SCC-DFTB. J. Chem. Theory Comput. 2011, 7, 931-948.

(25) Jin, H.; Goyal, P.; Das, A. K.; Gaus, M.; Meuwly, M.; Cui, Q. Copper Oxidation/Reduction in Water and Protein: Studies with DFTB3/MM and VALBOND Molecular Dynamics Simulations. J. Phys. Chem. B 2016, 120, 1894-1910. 
(26) Zhang, C.; Zhang, F.; Zhou, P.; Zhang, C. Functional role of metalloproteins in genome stability. Front. Biol. 2016, 11, 119-131.

(27) Solomon, E. I.; Szilagyi, R. K.; DeBeer George, S.; Basumallick, L. Electronic Structures of Metal Sites in Proteins and Models : Contributions to Function in Blue Copper Proteins. Chem. Rev. 2004, 104, 419-458.

(28) Pufahl, R. A.; Singer, C. P.; Peariso, K. L.; Lin, S.-J.; Schmidt, P. J.; Fahrni, C. J.; Culotta, V. C.; Penner-Hahn, J. E.; O'Halloran, T. V. Metal Ion Chaperone Function of the Soluble $\mathrm{Cu}(\mathrm{I})$ Receptor Atx1. Science 1997, 278, 853-856.

(29) Hazes, B.; Kalk, K. H.; Hol, W. G.; Magnus, K. A.; Bonaventura, C.; Bonaventura, J.; Dauter, Z. Crystal structure of deoxygenated limulus polyphemus subunit II hemocyanin at $2.18 \AA$ resolution: Clues for a mechanism for allosteric regulation. Prot. Sci. 1993, 2, 597-619.

(30) Noyori, R.; Kitamura, M. Enantioselective Catalysis With Metal-Complexes - an Overview. Modern Synthetic Methods. 1989; pp 115-198.

(31) Eckenhoff, W. T.; Pintauer, T. Structural Comparison of Copper(I) and Copper(II) Complexes with Tris(2-pyridylmethyl)amine Ligand. Inorg. Chem. 2010, 49, 1061710626.

(32) Cobeljic, B.; Pevec, A.; Turel, I.; Spasojevic, V.; Milcic, M.; Mitic, D.; Sladic, D.; Andelkovic, K. Analysis of the structures of the $\mathrm{Cu}(\mathrm{I})$ and $\mathrm{Cu}(\mathrm{II})$ complexes with 3acetylpyridine and thiocyanate. Polyhedron 2014, 69, 77-83.

(33) Das, A. K.; Meuwly, M. Hydration Control Through Intramolecular Degrees of Freedom: Molecular Dynamics of [Cu(II)(Imidazole) 4 ]. J. Phys. Chem. B 2017, 121, 9024-9031.

(34) Deeth, R. The ligand field molecular mechanics model and the stereoelectronic effects of d and s electrons. Coord. Chem. Rev. 2001, 212, 11-34. 
(35) Root, D. M.; Landis, C. R.; Cleveland, T. Valence bond concepts applied to the molecular mechanics description of molecular shapes. 1. Application to nonhypervalent molecules of the P-block. J. Am. Chem. Soc. 1993, 115, 4201-4209.

(36) Cleveland, T.; Landis, C. R. Valence Bond Concepts Applied to the Molecular Mechanics Description of Molecular Shapes. 2. Applications to Hypervalent Molecules of the P-Block. J. Am. Chem. Soc. 1996, 118, 6020-6030.

(37) Landis, C. R.; Cleveland, T.; Firman, T. K. Valence Bond Concepts Applied to the Molecular Mechanics Description of Molecular Shapes. 3. Applications to Transition Metal Alkyls and Hydrides. J. Am. Chem. Soc. 1998, 120, 2641-2649.

(38) Firman, T. K.; Landis, C. R. Valence bond concepts applied to the molecular mechanics description of molecular shapes. 4. Transition metals with pi-bonds. J. Am. Chem. Soc. 2001, 123, 11728-11742.

(39) Weinhold, F.; Landis, C. Valency and Bonding: A Natural Bond Orbital DonorAcceptor Perspective; Cambridge University Press, 2005.

(40) Tubert-Brohman, I.; Schmid, M.; Meuwly, M. Molecular Mechanics Force Field for Octahedral Organometallic Compounds with Inclusion of the Trans Influence. J. Chem. Theory Comput. 2009, 5, 530-539.

(41) Szymczak, J. J.; Hofmann, F. D.; Meuwly, M. Structure and dynamics of solvent shells around photoexcited metal complexes. Phys. Chem. Chem. Phys. 2013, 15, 6268-6277.

(42) Das, A. K.; Solomon, R. V.; Hofmann, F.; Meuwly, M. Inner-Shell Water Rearrangement Following Photoexcitation of Tris(2,2 '-bipyridine)iron(II). J. Phys. Chem. B 2016, 120, 206-216.

(43) van Duin, A.; Dasgupta, S.; Lorant, F.; Goddard, W. ReaxFF: A reactive force field for hydrocarbons. J. Phys. Chem. A 2001, 105, 9396-9409. 
(44) Warshel, A.; Weiss, R. M. An empirical valence bond approach for comparing reactions in solutions and in enzymes. J. Am. Chem. Soc. 1980, 102, 6218-6226.

(45) Schmitt, U.; Voth, G. Multistate empirical valence bond model for proton transport in water. J. Phys. Chem. B 1998, 102, 5547-5551.

(46) Maupin, C.; Wong, K.; Soudackov, A.; Kim, S.; Voth, G. A multistate empirical valence bond description of protonatable amino acids. J. Phys. Chem. A 2006, 110, 631-639.

(47) Nutt, D. R.; Meuwly, M. Studying reactive processes with classical dynamics: Rebinding dynamics in MbNO. Biophys. J. 2006, 90, 1191-1201.

(48) Danielsson, J.; Meuwly, M. Atomistic simulation of adiabatic reactive processes based on multi-state potential energy surfaces. J. Chem. Theory Comput. 2008, 4, 1083-1093.

(49) Nagy, T.; Yosa Reyes, J.; Meuwly, M. Multisurface Adiabatic Reactive Molecular Dynamics. J. Chem. Theory Comput. 2014, 10, 1366-1375.

(50) Magnusson, E. Hypercoordinate Molecules of $2^{\text {nd }}$-Row Elements - $d$-Functions or $d$ Orbitals. J. Am. Chem. Soc. 1990, 112, 7940-7951.

(51) Pimentel, G. The Bonding of Trihalide and Bifluoride Ions by the Molecular Orbital Method. J. Chem. Phys. 1951, 19, 446-448.

(52) Rundle, R. Probable Structure of $\mathrm{XeF}_{4}$ and $\mathrm{XeF}_{2}$. J. Am. Chem. Soc. 1963, 85, 112113.

(53) Coulson, C. Nature of Bonding in Xenon Fluorides + Related Molecules. J. Chem. Soc $1964,1442-\&$.

(54) Landis, C. R.; Weinhold, F. 3c/4e (sigma)over-cap-Type Long-Bonding: A Novel Transitional Motif toward the Metallic De localization Limit. Inorg. Chem. 2013, 52, 51545166. 
(55) Braida, B.; Hiberty, P. C. Application of the Valence Bond Mixing Configuration Diagrams to Hypervalency in Trihalide Anions: A Challenge to the Rundle-Pimentel Model. J. Phys. Chem. A 2008, 112, 13045-13052.

(56) Glowacki, D. R.; Orr-Ewing, A. J.; Harvey, J. N. Non-equilibrium reaction and relaxation dynamics in a strongly interacting explicit solvent: $\mathrm{F}+\mathrm{CD} 3 \mathrm{CN}$ treated with a parallel multi-state EVB model. J. Chem. Phys. 2015, 143.

(57) Pauling, L. The Nature of the Chemical Bond. Application of Results Obtained From The Quantum Mechanics and From a Theory of Paramagnetic Susceptibility to the Structure of Molecules. J. Am. Chem. Soc. 1931, 53, 1367-1400.

(58) Rappé, A.; Pietsch, M.; Wiser, D.; Hart, J.; Bormann-Rochotte, L.; Skiff, W. Rff, Conceptual Development of a Full Periodic Table Force Field for Studying Reaction Potential Surfaces. Molecular Engineering 1997, 7, 385-400.

(59) Pauling, L. The Nature of the Chemical Bond and the Structure of Molecules and Crystals: An Introduction to Modern Structural Chemistry; George Fisher Baker NonResident Lecture Series; Cornell University Press, 1960.

(60) Pauling, L. Correlation of nonorthogonality of best hybrid bond orbitals with bond strength of orthogonal orbitals. Proc. Natl. Acad. Sci. U. S. A. 1976, 73, 274-275.

(61) Appleton, T.; Clark, H.; Manzer, L. The trans-influence: its measurement and significance. Coordination Chemistry Reviews 1973, 10, 335 - 422.

(62) Jahn, H. A.; Teller, E. Stability of Polyatomic Molecules in Degenerate Electronic States. I. Orbital Degeneracy. Proceedings of the Royal Society of London A: Mathematical, Physical and Engineering Sciences 1937, 161, 220-235.

(63) Cisplatin. The American Society of Health-System Pharmacists. 2016, 
(64) Kelland, L. The Resurgence of Platinum-Based Cancer Chemotherapy. Nat. Rev. Cancer 2007, 7, 573-584.

(65) Bathgate, R.; Moelwynhughes, E. The Kinetics of Certain Ionic Exchange Reactions of The 4 Methyl Halides in Aqueous Solution. J. Chem. Soc. 1959, 2642-2648.

(66) Pedraza-Gonzalez, L.; Galindo, J. F.; Gonzalez, R.; Reyes, A. Revisiting the Dielectric Constant Effect on the Nucleophile and Leaving Group of Prototypical Backside $\mathrm{S}_{\mathrm{N}} 2$ Reactions: A Reaction Force and Atomic Contribution Analysis. J. Phys. Chem. A 2016, 120, 8360-8368.

(67) Bash, P.; Field, M.; Karplus, M. Free-Energy Perturbation Method for chemicalReactions in the Condensed Phase - a Dynamical-Approach Based on a Combined Quantum and Molecular Mechanics Potential. J. Am. Chem. Soc. 1987, 109, 80928094 .

(68) Purvis, G.; Bartlett, R. A Full Coupled-Cluster Singles and Doubles Model - the Inclusion of Disconnected Triples. J. Chem. Phys. 1982, 76, 1910-1918.

(69) Dunning, T. Gaussian-Basis Sets for Use in Correlated Molecular Calculations .1. The Atoms Boron Through Neon and Hydrogen. J. Chem. Phys. 1989, 90, 1007-1023.

(70) Jorgensen, W. L.; Chandrasekhar, J.; Madura, J.; Impey, R.; Klein, M. J. Chem. Phys. 1983, 79, 926.

(71) Muetterties, E.; Phillips, W. Structure of ClF3 and Exchange Studies on Some Halogen Fluorides by Nuclear Magnetic Resonance. J. Am. Chem. Soc. 1957, 79, 322-326.

(72) Hannon, M. J. Metal-Based Anticancer Drugs: From a Past Anchored in Platinum Post-Genomic Future and Biology. Pure Appl. Chem. 2007, 79, 2243-2261.

(73) Ting, V. P.; Schmidtmann, M.; Wilson, C. C.; Weller, M. T. Cisplatin: Polymorphism 
and Structural Insights into an Important Chemotherapeutic Drug. Angew. Chem. Int. Ed. 2010, 49, 9408-9411.

(74) Becke, A. D. Density-Functional Thermochemistry. III. The Role of Exact Exchange. J. Chem. Phys. 1993, 98, 5648-5652.

(75) Hay, P. J.; Wadt, W. R. Ab initio effective core potentials for molecular calculations potentials for the transition-metal atoms Sc to Hg. J. Chem. Phys. 1985, 82, 270-283.

(76) Hehre, W. J.; Ditchfield, R.; Pople, J. A. Self-Consistent Molecular Orbital Methods. XII. Further Extensions of Gaussian-Type Basis Sets for Use in Molecular Orbital Studies of Organic Molecules. J. Chem. Phys. 1972, 56, 2257-2261.

(77) Bates, D.; Watts, D. Nonlinear regression analysis and its applications; Wiley series in probability and mathematical statistics; Wiley: New York, 1988.

(78) Reed, A. E.; Weinstock, R. B.; Weinhold, F. Natural Population Analysis. J. Chem. Phys. 1985, 83, 735-746.

(79) A. D. MacKerell Jr.,; Bashford, D.; Bellott, M.; R. L. Dunbrack Jr.,; Evenseck, J. D.; Field, M. J.; Fischer, S.; Gao, J.; Guo, H.; Ha, S. et al. All-Atom Empirical Potential for Molecular Modeling and Dynamics Studies of Proteins. J. Phys. Chem. B 1998, 102, 3586-3616.

(80) Georgieva, I.; Trendafilova, N.; Dodoff, N.; Kovacheva, D. DFT Study of the Molecular and Crystal Structure and Vibrational Analysis of Cisplatin. Spectrochim. Acta Mol. Biomol. Spectrosc. 2017, 176, 58-66.

(81) Bell, C.; Price, N.; Chakrabarti, B. The Methyl Bromide Issue; Wiley Series in Agrochemicals \& Plant Protection; Wiley, 1996.

(82) Bulathsinghala, A. T.; Shaw, I. C. The Toxic Chemistry of Methyl Bromide. Hum. Exp. Toxicol 2014, 33, 81-91. 
(83) Adamovic, I.; Gordon, M. Solvent Effects on the $\mathrm{S}_{N} 2$ Reaction: Application of the Density Functional Theory-Based Effective Fragment Potential Method. J. Phys. Chem. A 2005, 109, 1629-1636.

(84) Pagliai, M.; Raugei, S.; Cardini, G.; Schettino, V. Car-Parrinello Molecular Dynamics on the $\mathrm{S}_{N} 2$ Reaction $\mathrm{Cl}^{-}+\mathrm{CH}_{3} \mathrm{Br}$ in Water. J. Mol. Struct. Theochem. 2003, 630, $141-149$.

(85) Bogdanov, B.; McMahon, T. Thermochemistry and Structures of Solvated $\mathrm{S}_{N} 2$ Complexes and Transition States in the Gas Phase: Experiment and Theory. Int. J. Mass Spectrom. 2005, 241, 205-223.

(86) Liu, P.; Li, C.; Wang, D. Multilevel Quantum Mechanics Theories and Molecular Mechanics Calculations of the $\mathrm{Cl}^{-}+\mathrm{CH}_{3}$ I Reaction in Water. J. Phys. Chem. A 2017, 121, 8012-8016.

(87) Li, C.; Ross, P.; Szulejko, J.; McMahon, T. High-pressure mass spectrometric investigations of the potential energy surfaces of gas-phase $\mathrm{S}_{N} 2$ reactions. J. Am. Chem. Soc. 1996, 118, 9360-9367.

(88) Schmatz, S.; Botschwina, P.; Stoll, H. Coupled cluster calculations for the $\mathrm{S}_{N} 2$ reaction $\mathrm{Cl}^{-}+\mathrm{CH}_{3} \mathrm{Br} \rightarrow \mathrm{ClCH}_{3}+\mathrm{Br}^{-}$. Int. J. Mass Spectrom. 2000, 201, 277-282.

(89) Kottalam, J.; Case, D. A. Dynamics of Ligand escape from the Heme pocket of Myoglobin. J. Am. Chem. Soc. 1988, 110, 7690-7697.

(90) Kumar, S.; Rosenberg, J. M.; Bouzida, D.; Swendsen, R. H.; Kollman, P. A. The Weighted Histogram Analysis Method for Free-Energy Calculations on Biomolecules. I. The Method. J. Compt. Chem. 1992, 13, 1011-1021.

(91) Soloviov, M.; Das, A. K.; Meuwly, M. Structural Interpretation of Metastable States in Myoglobin-NO. Angew. Chem. Int. Ed. 2016, 55, 10126-10130. 